\title{
VIBRATION ANALYSIS AND DEFORMATION MEASUREMENT
}

\author{
H. J. Tiziani \\ Wild Heerbrugg Ltd. \\ Heerbrugg \\ Switzerland
}

5.1 Visual Detection of Vibration 73

5.1.1 Vibration Detection in the Presence of a Reference Wave 74

5.1.2 Vibration Detection without a Reference Field 75

5.2 Deformation Measurement by Speckle Interferometry 77

5.3 In-Plane Oscillations by Image Plane Recording 79

$\begin{array}{ll}\text { 5.3.1 Theory } & 80\end{array}$

5.3.2 Time-Average Exposures of in-Plane Oscillations 83

$\begin{array}{lll}\text { 5.3.3 Display of Young's Fringes } & 84\end{array}$

5.3.4 Comparison of Fringe Shapes for Different Motions 89

5.4 Tilt Analysis by Fourier Transform Plane Recording 92

5.4.1 Two Successive Exposures 94

5.4.2 Multiple Successive Exposures 97

5.4.3 Two Successive Exposures with Two Beam Illumination 99

5.5 Limitations of Speckle Photography for Engineering Measurements 101

$\begin{array}{ll}\text { 5.5.1 Movements Parallel to the Line-of-Sight } & 101\end{array}$

$\begin{array}{ll}\text { 5.5.2 Limits on Tilts for in-Plane Measurements } & 102\end{array}$

$\begin{array}{ll}\text { 5.5.3 Coherence of the Laser Source } & 103\end{array}$

$\begin{array}{lll}5.6 \text { Discussion } & 104\end{array}$

$\begin{array}{ll}\text { 5.6.1 Tilt and Deformation Measurement } & 104\end{array}$

$\begin{array}{ll}\text { 5.6.2 Vibration Measurement } & 105\end{array}$

$\begin{array}{ll}\text { 5.6.3 Deformation and White Light Speckle Photography } & 108\end{array}$

$\begin{array}{ll}\text { References } & 109\end{array}$

\subsection{Visual Detection of Vibration}

An early utilization of laser speckle was the detection of nodal lines of movements parallel to the line of sight (see, for example, [5.1-5.3]). The resulting patterns resembled the classical Chladni sand patterns. Real time optical methods for vibration analysis rely on speckles produced by laser illumination of an optically rough surface. The main function of the visual speckle interferometer is the detection of vibrations and the map- 
ping of nodal lines. If an object surface is moving in and out with an amplitude on the order of one-quarter wavelength or more, its speckle pattern will become blurred out. Further, its visible texture will alter. If the object is moving fast enough, the contrast of the speckles will become very poor. However, for stationary areas of the surface, such as the nodal regions, the eye will see high-contrast speckle patterns.

\subsubsection{Vibration Detection in the Presence of a Reference WAVE}

The original speckle interferometer, shown schematically in Fig. 5.1, was developed by Archbold et al. [5.1], and subsequently perfected by Stetson [5.4]. The telescope for sighting the object is equipped with a small adjustable iris diaphragm at its entrance pupil which governs the speckle size observed. A reference beam is introduced near the image plane of the object by means of a beam splitter in the shape of a wedge. This permits a separation of the two reflected beams (from the back and front surfaces) in order that one can be eliminated by an additional diaphragm located near the observer's eye. For high-contrast fringes, a polarizing filter can be mounted in front of the telescope and oriented so that only light from the object, polarized in the same plane as the reference beam, is transmitted. The object and reference beams originate from the same laser source, thereby providing the necessary coherence. For a laser with limited coherence length, the optical path lengths of the two beams must be almost equal.

According to Burch [5.5], an interference pattern formed between a speckle pattern and a uniform reference wave will have a brightness distribution largely dependent on the mutual coherence between the wavefronts. Thus, for stationary parts of the surface the coherence is high,

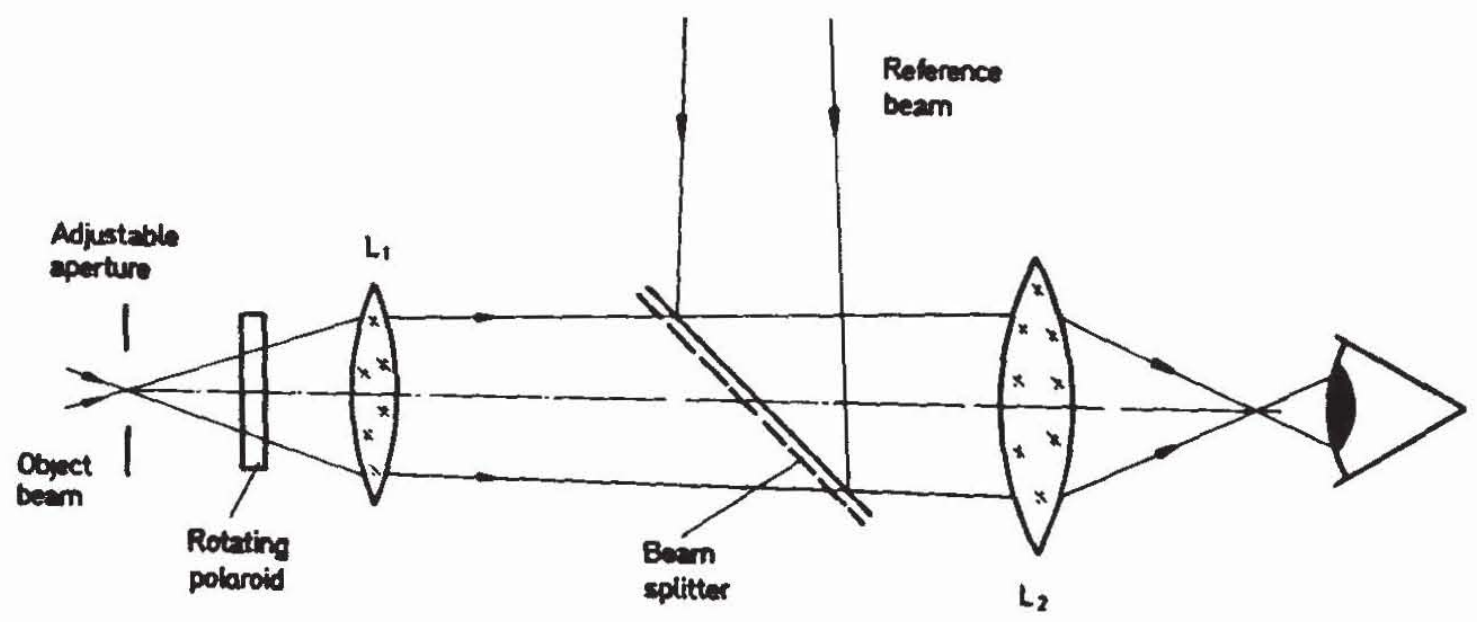

FIG. 5.1 Optical system for visual speckle interferometry with a reference wave. 
resulting in a highly speckled image with good contrast. For vibrating regions, the coherence is reduced because the eye integrates the brightness variations over the vibration cycle, resulting in a smoother intensity distribution. For the greatest visual sensitivity, the reference beam is usually chosen to be brighter than the signal beam, and can have a value almost four times the average speckle brightness. The sensitivity to object motions occurring parallel to the line of sight is that of a corresponding time-average holographic recording [5.6]. The dynamic range is, however, rather limited. But evaluation can be made in real time. The speckle interferometer is, therefore, a valuable complement to the real time holographic interferometer. A slow change in the position of any object placed in the field of view of a speckle interferometer may be detected by the twinkling appearance of the speckles. Therefore, the interferometer can also be a very useful tool for deciding whether an object under study is creeping or vibrating, or whether air currents are affecting the optical path. However, for quantitative analyses an additional holographic technique may be useful.

The blurring effect of vibration is related to the amplitude of the motion. Ek and Molin [5.2] considered the statistics of the brightness variation of the speckles and showed that their contrast $C$ varies with the vibration amplitude $\rho$ according to the equation

$$
\mathrm{C}=\left[1+2 \alpha J_{0}^{2}(4 \pi \rho / \lambda)\right]^{1 / 2} /(1+\alpha) .
$$

The illumination and viewing are assumed to be almost normal to the surface, $\alpha$ is the ratio of the reference beam intensity to that of the object beam, and $J_{0}$ is the Bessel function of order zero and the first kind. This equation predicts that the contrast will periodically fall to a minimum with a decreasing amplitude. This is a result similar to that obtained by vibration analysis using holographic interferometry [5.6]. For a well-adjusted visual speckle interferometer it is possible to distinguish the first, and sometimes the second, subsidiary maximum of speckle contrast. Video techniques have also been applied to the problem of speckle vibration analysis as described by Butters in Chapter 6 .

\subsubsection{Vibration Detection without a Reference Field}

A method to detect nodal lines without using a reference wave, but with a diffuser in the illuminating laser beam, was described by Eliasson and Mottier [5.3]. The technique applies only to the detection of nodal areas, but the sensitivity of the method to the vibration amplitude can be changed by using different diffusers, or by varying the distance between the object and the diffuser. As seen in the experimental setup (Fig. 5.2) a 


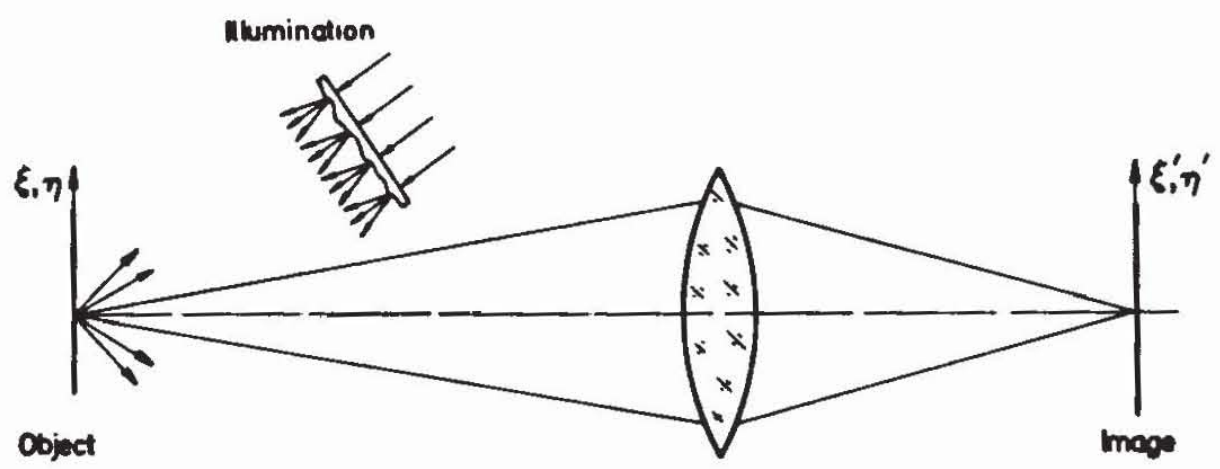

Fig. 5.2 Principle of visual speckle interferometry without a reference wave.

laser illuminates the object through a diffuser, providing almost uniform illumination. An image of the object is formed by a camera lens, and the difference between stationary and moving object areas lies in the timeaveraged contrast of the speckles. For an area at rest this contrast is nearly unity and thus nodal lines appear with high contrast. For increasing vibration amplitude the contrast gradually decreases until it eventually vanishes for large amplitudes.

In addition to direct observation, the speckle pattern of the vibrating object can also be photographed. However, for recording speckle contrast, a conventional photograph of the vibrating object is very often not satisfactory because the dynamic range of the photographic material is too narrow to record the bright spots and the dark patches in the nodal region linearly. Therefore it is difficult to distinguish between object areas at rest and adjacent ones with small amplitudes of vibration. However, the nonlinearity of photographic emulsions can be taken into account. Speckle intensities above average are clipped due to the saturation of the emulsion, and intensities lower than the average are imaged quasilinearly. Therefore, applying a spatial filter to enhance only the high spatial frequencies resulting from the nodal lines improves the technique. A result obtained by Eliasson and Mottier [5.3] is presented in Fig. 5.3A, where the speckles remain distinct for stationary regions and reproduce the nodal lines. The object was oscillated at a frequency of $2 \mathrm{kHz}$ normal to the surface, and it is apparent that a quantitative analysis of the amplitude of vibration would be difficult. (Eliasson and Dändliker have discussed the contrast of the photographically recorded speckle pattern in detail [5.7].) A result of time-average holography for the same object is shown in Fig. $5.3 \mathrm{~B}$, from which an analysis would give the amplitude of oscillation. Although the described speckle technique gives general information about the vibration, the analysis depends very much on the photographic process. 

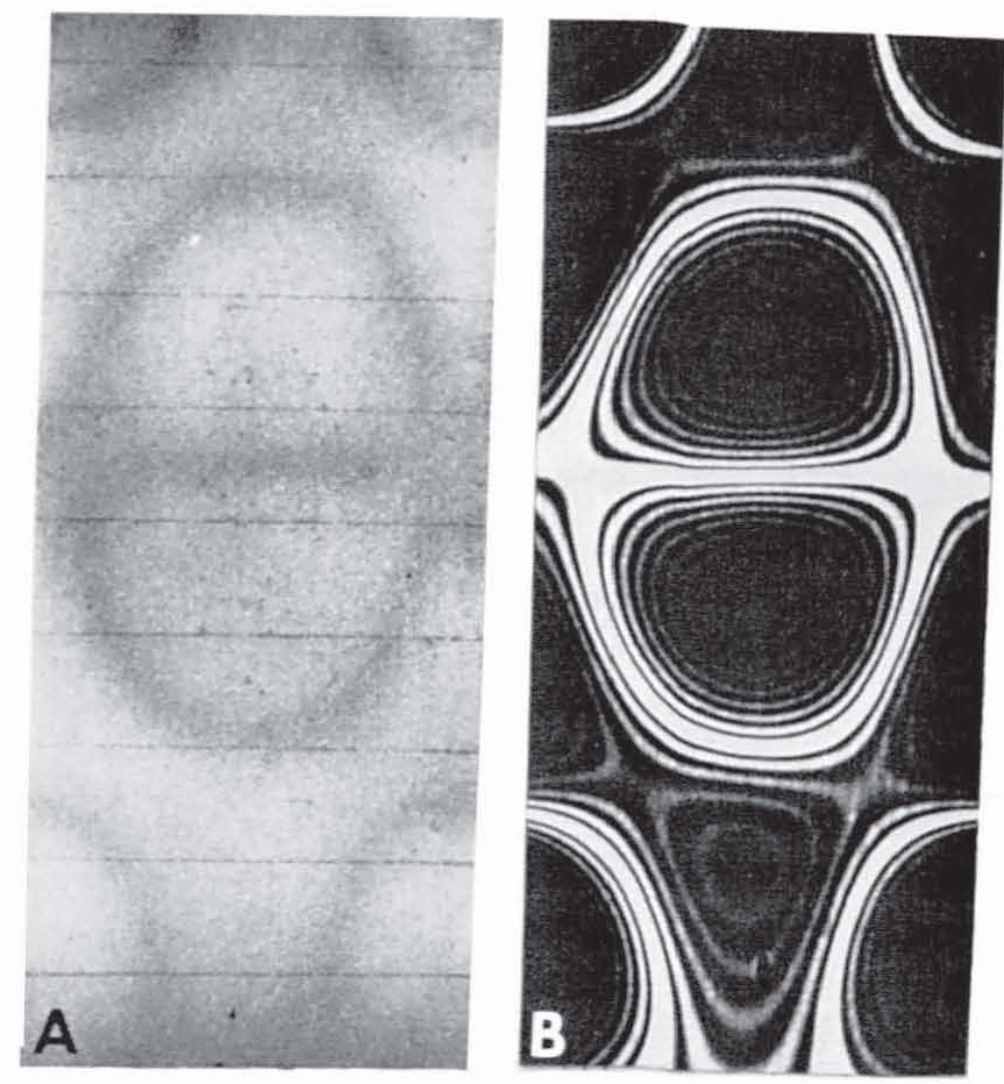

FIG. 5.3 Vibration patterns of a turbine blade from time-average recordings obtained (A) with a speckle interferometer and (B) holographically. (From Eliasson and Mottier [5.3].)

\subsection{Deformation Measurement by Speckle Interferometry}

Normal, as well as in-plane, displacements can be measured by causing two speckle patterns to interfere. The initial paper on the topic was presented by Leendertz in 1969 and subsequently published [5.8]. (Groh [5.9] had used the speckle effect to detect vibration-induced fatigue.) It became customary usage to refer to speckle interferometry if two waves were superposed. An equivalent optical interferometer can usually be readily modified for speckle interferometry, whereas for speckle photography such a construction would be difficult. In speckle interferometry, fringes are formed by fluctuation in correlation, or by matching of the two recorded speckle patterns.

Visual speckle interferometers are often linked to a Michelson interferometer with one of the mirrors replaced by an optically rough surface. Leendertz [5.8] replaced both of the mirrors by diffusely reflecting surfaces as shown in Fig. 5.4. If the laser light, which illuminates the interferometer, is linearly polarized and is not depolarized by diffuse reflections, a well-modulated speckle pattern is obtained at the image formed by the lens. Its character remains basically the same whether one, the other, 


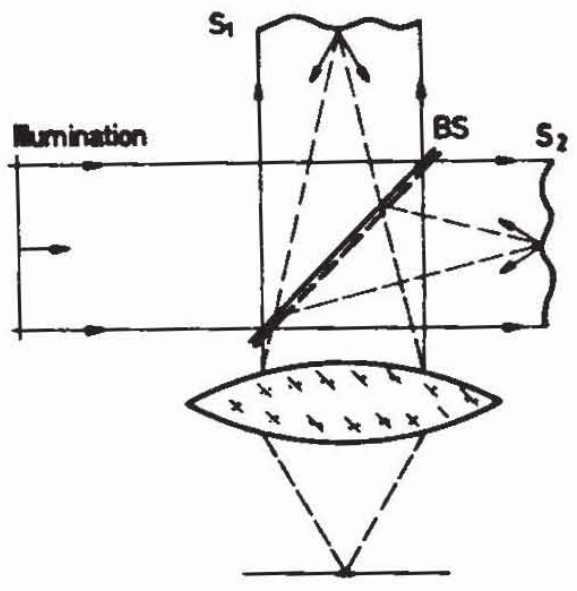

FIG. 5.4 Speckle pattern interferometer combining two speckle fields $\left(S_{1}\right.$ and $\left.S_{2}\right)$ with a beam splitter (BS).

or both diffuse surfaces are present. If one surface is moved in the direction of its normal, so that the phase of the light scattered from it changes everywhere by an amount $\Delta$, the combined field will differ from the original one unless the phase difference is a multiple of $2 \pi$ (i.e., $\Delta=k 2 \pi$; $k=1,2, \ldots K)$. In this case the speckle pattern will be the same as the original one. This can be considered as the interference of two mutually coherent speckle fields. The displaced diffuser changes the speckle field. The indicated phase is, of course, added in bulk to the random phase distribution of the speckled fields. When the relative phase is $\pi$, or an odd multiple thereof, the resulting field will be randomly different.

It is possible to detect positions of correlation (i.e., positions where the optical path difference is a multiple or $2 \pi$ ). This leads to a technique for measuring phase changes or surface movements. One method, though not the most efficient, is to record the speckle pattern (image of the diffuser) on a photographic plate. After processing, the plate is positioned in its original place. As a result of photographically processing the negative, dark areas will correspond to bright speckles and clear areas to dark ones. Low light transmission will therefore be obtained for matching speckle patterns (i.e., when the phase difference is zero or a multiple of $2 \pi$ ). Furthermore, the nonlinearities of the photographic emulsion can be successfully used. If the combined field change is not equal to a multiple of $2 \pi$, light will be transmitted through the plate because the new speckle field and the mask do not match one another. In addition to the measurement parallel to the optical axis of the entire optically rough surface, local displacement in the same direction or small tilts can be detected. Dark fringes occur when part of the object is out of phase by a multiple of $2 \pi$.

Similar results can be obtained by utilizing the double exposure technique and recording speckle patterns before and after deformation. In the zones where the speckles match, bright fringes will occur and the region will appear fairly transparent. In the zones where they do not match, 
superposition of the bright speckles of one pattern on the dark speckles of the other will, when combined with overdevelopment, result in low transmittance. The result will be light and dark bands in the photograph corresponding exactly to the light and dark fringes obtainable if the diffuse surfaces were replaced by plane mirrors one of which was given the same movement.

The photographic transparency with the double exposed speckle patterns could be bleached into a silver halide. The regions of matching speckle will be very effective in diffracting light that passes through the transparency relative to areas where the speckle patterns do not match. An optical processing system can be used to detect this by placing a camera on axis and blocking off the zero-order beam. Only light diffracted away from the optical axis is accepted.

The low-intensity fringe patterns produced by the photographic mask method have to be photographed from the interferometer in real time. The contrast of the fringes obtained may be poor and the application is very limited. Leendertz $[5.8,5.10]$ recognized, however, that the two speckle fields could be derived from the same object by illuminating the optically rough surface with two mutually coherent laser beams, as discussed in the previous chapter (see Fig. 4.1). The relative phase of the two speckled fields will change only for motions of the object parallel to the vector difference of the propagation of the two fields, i.e., the method is sensitive only for displacements perpendicular to the fringe pattern produced by the two plane waves. If the two beams make equal angles with the surface normal of the object, this direction lies in the plane of the object. Thus, fringes obtained in this configuration will measure variations in the inplane motions of the surface. They are suitable for measuring in-plane surface strains, as considered in detail in Chapter 4.

In laser speckle interferometry the stability required is usually less severe than in real time holographic interferometry. Stability is needed only for a few seconds and is determined by the persistence and visualizing time necessary for evaluation. The same types of studies and measurements are possible by means of laser speckle interferometry as are possible by real time hologram interferometry.

\subsection{In-Plane Oscillations by Image Plane Recording}

Interest in speckle application has grown considerably during the last few years. Double exposure speckle pattern applications have played an important role in this development, and have proved to be very useful for deformation and vibration analysis. For the following applications it is appropriate to record the speckle pattern either in the image plane (as 
described in this section) or in the Fourier transform plane (as described in Section 5.4). For in-plane movements the image plane is generally used, while for tilt analysis the Fourier plane is appropriate.

\subsubsection{THEORY}

An optically rough surface illuminated with coherent light leads to speckle formation in space, including the image plane. For our purposes, the analysis of the speckles recorded in the image plane will be carried out by image formation of an optically rough surface illuminated by coherent light such as that from a laser. The optically rough surface may be described by its spectrum [5.11]. Thus, image formation with coherent light is discussed below in order to analyze speckling for deformation and vibration analysis.

The wavefront immediately after the object may be written as

$$
A(\xi)=\Phi(\xi) A_{\mathrm{o}}(\xi)
$$

where $A_{0}(\xi)$ includes the object structure and the illumination. The optically rough surface may be described by the superposition of a set of waves, namely

$$
\Phi(\xi)=\sum_{p} \psi\left(x_{p}\right) \exp \left[i 2 \pi x_{p} \cdot \xi / \lambda R\right],
$$

which may be written as an integral in the limit to represent the distribution of complex amplitudes as a function of $\xi$ by its Fourier integral. $\xi$ and $\mathbf{x}_{p}$ are two-dimensional vectors, representing the rectangular coordinates $(\xi, \eta)$ in the object plane and $\left(x_{p}, y_{p}\right)$ in the pupil plane. It can be shown that a Fourier transform relationship exists between the complex amplitude distribution of the object and entrance pupil, as well as between the exit pupil and the image [5.12]. Image formation is therefore a two-step procedure, namely, diffraction between the object and entrance pupil, and between the exit pupil and image (see Fig. 5.5). The transfer from a complex amplitude in the entrance to the exit pupil is obtained by multiplication by the pupil function $f(x, y)$, which takes into account the quality of the image-forming system. The complex amplitude on the entrance pupil sphere, with its center of curvature in the object plane, can be written as

$$
a(x, y)=K \iint_{-\infty}^{\infty} A(\xi, \eta) \exp \left[\frac{i 2 \pi(\xi x+\eta y) n}{\lambda R}\right] d \xi d \eta,
$$

where $A(\xi, \eta)$ is the complex amplitude immediately in front of the object. According to customary usage [5.12], reduced coordinates for image formation are used, namely: 


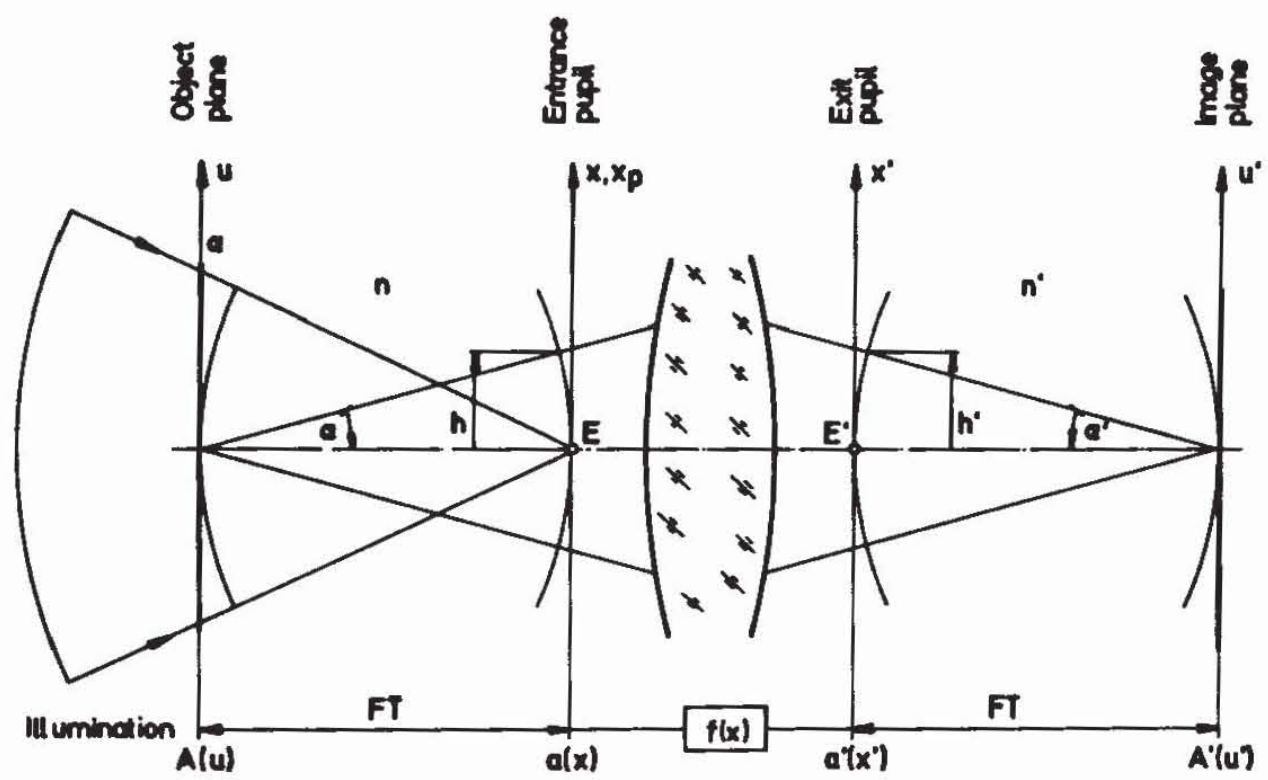

FIG. 5.5 Schematic diagram to illustrate the principle of coherent image formation.

$$
\begin{array}{ccc}
u=(n \xi \sin \alpha) / \lambda & X_{p}=x_{p} / h & X=x / h \\
v=(n \eta \sin \alpha) / \lambda & Y_{p}=y_{p} / h & Y=y / h .
\end{array}
$$

The notations are as shown in Fig. 5.5 (one-dimensional):

$R=$ separation object-entrance pupil plane;

$\alpha, \alpha^{\prime}=$ aperture angle of the optical system in the object and image space respectively;

$n, n^{\prime}=$ refractive index in the object and image space;

$\lambda=$ wavelength in vacuum;

$\xi, \eta=$ rectangular coordinates in the object space;

$X_{p}, Y_{p}=$ normalized pupil coordinates;

$X, Y=$ normalized coordinates of the intersection $x, y$ of the finite rays with the pupil sphere;

$h, h^{\prime}=$ paraxial ray heights at the entrance and exit pupils respectively.

A similar notation will be used later for the exit pupil and image space. $K$ is a constant and will be neglected. The complex amplitude on the entrance pupil sphere, using the reduced coordinates, is the Fourier transform of $A(u, v)$, namely:

$$
a(X, Y)=\iint A(u, v) \exp [-i 2 \pi(u X+v Y)] d u d v .
$$

Substituting from Eq. (5-2), the total complex amplitude in the entrance pupil can be written by using vector notation as 


$$
a(\mathrm{X})=\iint \Phi(\mathrm{u}) A_{\mathrm{o}}(\mathrm{u}) \exp [-i 2 \pi \mathrm{u} \cdot \mathrm{X}] d \mathbf{u} .
$$

By integrating over the object area and considering the Fourier transform of $A_{0}(\mathrm{u})$ to be a $\delta$ function, $a(X)=\varphi(X)$, where $\varphi(X)$ is the Fourier transform of the optically rough surface. An additional tilt will lead to a lateral shift of the speckles in the pupil. For simplicity, the influence of tilts will be discussed separately, later. The displacement $\Delta u$ of an optically rough surface, or a part of it, leads to a phase shift of the speckle pattern, and its complex amplitude in the entrance pupil is

$$
a(\mathrm{X}, \Delta \mathrm{u})=\varphi(\mathrm{X}) \exp [i 2 \pi \Delta \mathrm{u} \cdot \mathbf{X}] .
$$

For a good optical system we can assume $\mathbf{X}=\mathbf{X}^{\prime}$, where $\mathbf{X}$ and $\mathbf{X}^{\prime}$ are the normalized entrance and exit pupil coordinates.

Considering an aberration-free system (i.e., $f(X)$ is constant over the pupil) the corresponding complex amplitude in the image is

$$
A^{\prime}\left(\mathrm{u}^{\prime}, \Delta \mathrm{u}\right)=\iint_{-\infty}^{\infty} f(\mathrm{X}) a(\mathrm{X}, \Delta \mathrm{u}) \exp \left[i 2 \pi \mathrm{u}^{\prime} \cdot \mathrm{X}^{\prime}\right] d \mathrm{X}^{\prime} .
$$

The integration is extended over the exit pupil of the optical system, where the pupil function of an ideal optical system $f(X, Y)$ is 1 for $\left(X^{2}+Y^{2}\right)^{1 / 2} \leqslant 1$, and 0 otherwise. Therefore, by substituting Eq. (5-7) into the above equation, we obtain $A^{\prime}\left(\mathrm{u}^{\prime}, \Delta \mathrm{u}\right)=\Phi^{\prime}\left(\mathrm{u}^{\prime}+\Delta \mathrm{u}\right)$, or, by substituting for $\Phi^{\prime}\left(\mathbf{u}^{\prime}\right)$, noticing that $\mathbf{u}^{\prime}$ and $\mathbf{u}$ are normalized coordinates defined in Eq. (5-5) and applying the sine condition $n \xi \sin \alpha=n^{\prime} \xi^{\prime} \sin \alpha^{\prime}$ we can write $\Delta \mathbf{u}^{\prime}=\Delta u$ (i.e., the magnification is taken care of by the normalization of the coordinates). The intensity in the image plane is

$$
\begin{aligned}
I^{\prime}\left(\mathbf{u}^{\prime}, \Delta \mathbf{u}^{\prime}\right) & =\left|A^{\prime}\left(\mathbf{u}^{\prime}, \Delta \mathbf{u}^{\prime}\right)\right|^{2} \\
& =\sum_{p} \sum_{q} \psi\left(\mathbf{X}_{p}\right) \psi^{*}\left(\mathbf{X}_{q}\right) \exp \left[i 2 \pi\left(\mathbf{X}_{p}-\mathbf{X}_{q}\right) \cdot\left(\mathbf{u}^{\prime}+\Delta \mathbf{u}^{\prime}\right)\right],
\end{aligned}
$$

leading to pairs of speckle patterns displaced laterally by $\Delta u^{\prime}$. The speckles in the image are shifted in exactly the same way as the image of the optically rough surface, provided additional tilts are small. The size of the speckles is governed by the size of the illuminated exit pupil. $\psi, \psi^{*}$ are zero outside the pupil [5.13]. By replacing the sums by integrals in the limit, and by substituting $\mathbf{X}_{r}$ for $\mathbf{X}_{p}-\mathbf{X}_{q}$, Eq. (5-8) can be writen

$$
I\left(\mathbf{u}^{\prime}, \Delta \mathbf{u}^{\prime}\right)=\iint\left\{\int \psi\left(\mathbf{X}_{r}+\mathbf{X}_{q}\right) \psi^{*}\left(\mathbf{X}_{q}\right) d \mathbf{X}_{q}\right\} \exp \left[i 2 \pi \mathbf{X}_{r} \cdot\left(\mathbf{u}^{\prime}+\Delta \mathbf{u}^{\prime}\right)\right] d \mathbf{X}_{r} .
$$

The first integral represents the autocorrelation of the illuminated exit pupil, and accounts for the size of the speckles. The second integral represents pairs of speckles (i.e., before and after displacement). 


\subsubsection{Time-Average Exposures of in-Plane Oscillations}

Not only lateral in-plane movements, but also lateral in-plane mechanical oscillations can be analyzed in this way. Speckle photography was found to be particularly useful for vibration analysis [5.11, 5.14]. For this application the intensity distribution in the image of an optically rough oscillating surface was recorded during a number of cycles, in order to study the time-averaged intensity distribution of the speckle pattern.

The amplitude of the in-plane oscillation of the object, or part of it, may be written as

$$
A_{1}(\mathrm{u}, \rho)=A_{0}(\mathrm{u}+\rho \cos 2 \pi \nu t) \Phi(\mathrm{u}+\rho \cos 2 \pi \nu t),
$$

where $\rho$ is the amplitude of the lateral oscillation in the object space. $A_{0}(u)$ includes the illumination and the object superposed on the opticallyrough surface, which for simplicity is considered to be unity. Normalized coordinates are used for $\rho$ in the same way as previously used for $u$. The intensity in the image can be written in accordance with Eq. (5-8) as

$$
\begin{aligned}
I\left(\mathbf{u}^{\prime}, \boldsymbol{\rho}, t\right)= & \sum_{\boldsymbol{p}} \sum_{q} \psi\left(\mathbf{X}_{p}\right) \psi^{*}\left(\mathbf{X}_{q}\right) \exp \left[i 2 \pi \mathbf{u}^{\prime} \cdot\left(\mathbf{X}_{p}-\mathbf{X}_{q}\right)\right] \\
& \times \exp \left[i 2 \pi\left(\mathbf{X}_{p}-\mathbf{X}_{q}\right) \cdot \rho \cos 2 \pi \nu t\right] .
\end{aligned}
$$

The time-average intensity is obtained by integration over time $t$. Applying Jacobi's theorem [5.15] for $t_{0} \gg 1 / \nu$, where $t_{0}$ is the response time of a typical detector (such as the exposure time of a photographic plate), we obtain

$$
\begin{aligned}
\left\langle I\left(\mathbf{u}^{\prime}, \boldsymbol{\rho}^{\prime}, t\right)\right\rangle= & \sum_{\mathbf{p}} \sum_{\mathbf{q}} \psi\left(\mathbf{X}_{p}\right) \psi^{*}\left(\mathbf{X}_{q}\right) J_{0}\left[2 \pi \boldsymbol{\rho}^{\prime} \cdot\left(\mathbf{X}_{p}-\mathbf{X}_{q}\right)\right] \\
& \times \exp \left[i 2 \pi \mathbf{u}^{\prime} \cdot\left(\mathbf{X}_{p}-\mathbf{X}_{q}\right)\right] .
\end{aligned}
$$

Changing coordinates $\mathbf{X}_{p}-\mathbf{X}_{q}=\mathbf{X}_{r}$ and replacing the sums by integrals, in the limit we can write

$$
\left\langle I\left(\mathbf{u}^{\prime}, \boldsymbol{\rho}^{\prime}, t\right)\right\rangle=\iint_{\times}\left[\iint_{\exp } \psi\left(i 2 \pi \mathbf{X}_{r}+\mathbf{X}_{q}\right) \mathbf{u}_{r}\right] d \mathbf{X}_{r} \text {. }
$$

The first integral represents the autocorrelation of the speckling in the exit pupil of the image-forming optical system, and the second part is similar to the result obtained by time-average holography [5.6]. It can be considered as the probability density function of a particular speckle, namely (for one-dimensional notation)

$$
\operatorname{rect}\left(u^{\prime} / \rho^{\prime}\right) /\left[\pi\left(u^{\prime 2}-\rho^{\prime 2}\right)^{1 / 2}\right] .
$$




\subsubsection{Display of Young's Fringes}

\subsubsection{Two Successive Exposures}

To observe Young's interference fringes, speckle patterns are most frequently recorded on photographic emulsions. Following exposure, before and after displacement, the two successive speckle patterns are processed and then illuminated by a coherent beam of light as shown, for instance, in Fig. 5.6a. The complex amplitude in the back focal plane of the lens $L_{1}$ for an object in its front focal plane is given by the Fourier transform of the recorded intensity, namely

$$
a\left(\mathrm{X}_{1}\right)=\iint T\left(\mathrm{u}^{\prime}, \Delta \mathrm{u}^{\prime}\right) \exp \left[-i 2 \pi \mathrm{u}^{\prime} \cdot \mathrm{X}_{1}\right] d \mathbf{u}^{\prime},
$$

where: $T\left(\mathrm{u}^{\prime}, \Delta \mathrm{u}^{\prime}\right)=T_{0}-b t_{0} I\left(\mathrm{u}^{\prime}, \Delta \mathrm{u}^{\prime}\right)$ is the approximate amplitude transparency of the developed photographic plate; $t_{0}$ is the exposure time; and $T_{0}$ and $b$ are constants of the photographic material. Using this relationship, the complex amplitude may be expressed as

$$
a\left(\mathbf{X}_{1}\right)=\iint\left\{T_{0}-b t_{0}\left[I^{\prime}\left(\mathbf{u}^{\prime}\right)+I^{\prime}\left(\mathbf{u}^{\prime}+\Delta \mathbf{u}^{\prime}\right)\right]\right\} \exp \left[-i 2 \pi \mathbf{u}^{\prime} \cdot \mathbf{X}_{1}\right] d \mathbf{u}^{\prime},
$$

where $\mathbf{X}_{1}=\mathbf{x}_{1} /\left(f_{1} \sin \alpha^{\prime}\right)$ are again normalized coordinates of the rectangular coordinates $\left(x_{1}, y_{1}\right)$ written as vectors and $f_{1}$ is the focal length of the lens performing the Fourier transform. For illuminating the developed speckle pattern by a plane (Fig. 5.6a) or spherical (Fig. 5.6b) wave, the diameter of the illuminating beam at the photographic plate should be large enough to obtain good fringe contrast. The speckles resulting from the limiting aperture will further reduce the contrast of the fringes obtained. For the analysis the limiting aperture will be neglected (although it will determine the speckle size in the fringe plane, which should be smaller than the fringe spacing). The central peak, resulting from $T_{0}$ in Eq. (5-13), and the constants will also be neglected to simplify the writing.

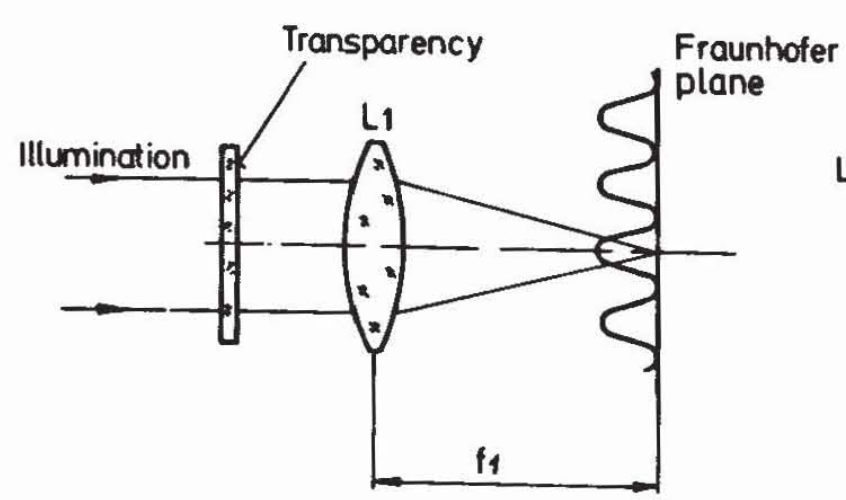

(a)

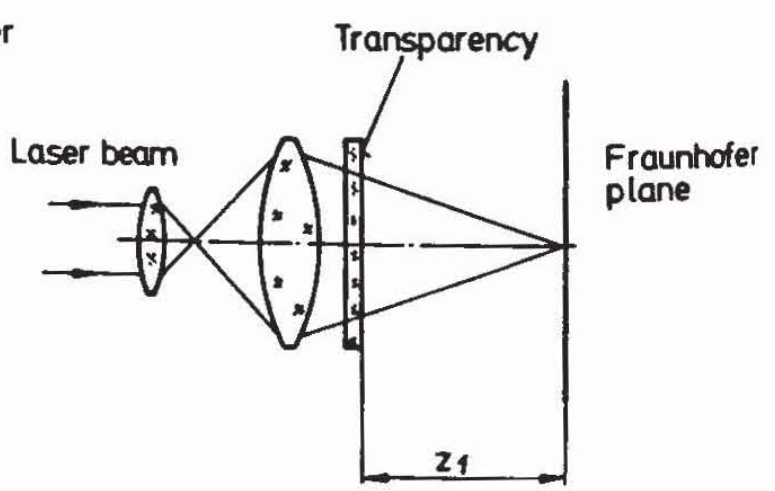

(b)

FiG. 5.6 Arrangements for displaying Young's fringes in the analysis of speckle pattern recordings. 
The amplitude in the back focal plane of the lens $L_{1}$ will be (by substituting from Eq. (5-8))

$$
\begin{aligned}
\tilde{a}\left(\mathbf{X}_{1}\right)= & \iint \sum_{\mathbf{p}} \sum_{\mathbf{q}} \psi\left(\mathbf{X}_{p}\right) \psi^{*}\left(\mathbf{X}_{q}\right) \exp \left[i 2 \pi \mathbf{u}^{\prime} \cdot\left(-\mathbf{X}_{1}+\mathbf{X}_{p}-\mathbf{X}_{q}\right)\right] \\
& \times d \mathbf{u}^{\prime}\left\{1+\exp \left[i 2 \pi\left(\mathbf{X}_{p}-\mathbf{X}_{q}\right) \cdot \Delta \mathbf{u}^{\prime}\right]\right\},
\end{aligned}
$$

which can be written in the limit, by substituting for $\mathbf{X}_{p}-\mathbf{X}_{q}=\mathbf{X}_{r}$, as

$$
\tilde{a}\left(\mathbf{X}_{1}\right)=\iint \psi\left(\mathbf{X}_{1}+\mathbf{X}_{q}\right) \psi^{*}\left(\mathbf{X}_{q}\right) d \mathbf{X}_{q}\left\{1+\exp \left[i 2 \pi \mathbf{X}_{1} \cdot \Delta \mathbf{u}^{\prime}\right]\right\} .
$$

The detector, such as a photographic emulsion or a photoelectric device, will record intensities only, and therefore

$$
\left|\tilde{a}\left(\mathbf{X}_{1}\right)\right|^{2}=2\left|\iint \psi\left(\mathbf{X}_{1}+\mathbf{X}_{q}\right) \psi^{*}\left(\mathbf{X}_{q}\right) d \mathbf{X}_{q}\right|^{2}\left\{1+\cos 2 \pi \mathbf{X}_{1} \cdot \Delta \mathbf{u}^{\prime}\right\} .
$$

The square of the autocorrelation of the pupil is modulated by Young's fringes, which are perpendicular to the direction of displacement. The interference fringes result from the superposition of the sums of two identical but laterally displaced speckle patterns recorded photographically. The two speckles of a pair act as small, identical, coherent sources and give the classical Young's fringes in the Fraunhofer diffraction plane. For an additional superposed object, the autocorrelation of the spectrum of the optically rough surface would have to be convolved with that of the object.

From Eq. (5-14) the in-plane displacement in the object is found to be

$$
|\Delta \xi|=\left(f_{1} \lambda\right) /\left(M\left|\mathbf{x}_{c}\right|\right),
$$

where $M$ is the lateral magnification of the image-forming system, $x_{c}$ the period of the fringes obtained in the Fraunhofer diffraction plane, and $f_{1}$ the focal length of the lens displaying the fringes or the distance from the plate or film to the Fraunhofer plane-when the plate is illuminated by a côverging beam, as shown in Fig. 5.6b. (Intensities only are observed.)

To demonstrate the technique, photographs of fringes resulting from the bending of a lever under load are shown in Fig. 5.7. A speckle pattern was recorded on a photographic plate before and after the load had been applied. A point-by-point analysis of the recorded speckled image produced the fringes depicted, from which the deformation, and its direction, can be deduced.

To observe the object deformation as a whole or the direction of movement, an additional small displacement (10-20 $\mu \mathrm{m}$, for example) is introduced between the two exposures. After development of the photographic plate, Young's fringes are obtained. With a slit placed in a dark fringe in the back focal plane of the lens $L_{1}$, which displays Young's fringes (Fig. 5.6), we can block the light coming from deformation-free regions. By contrast, deformed regions of the object produce fringes of different spacing and 


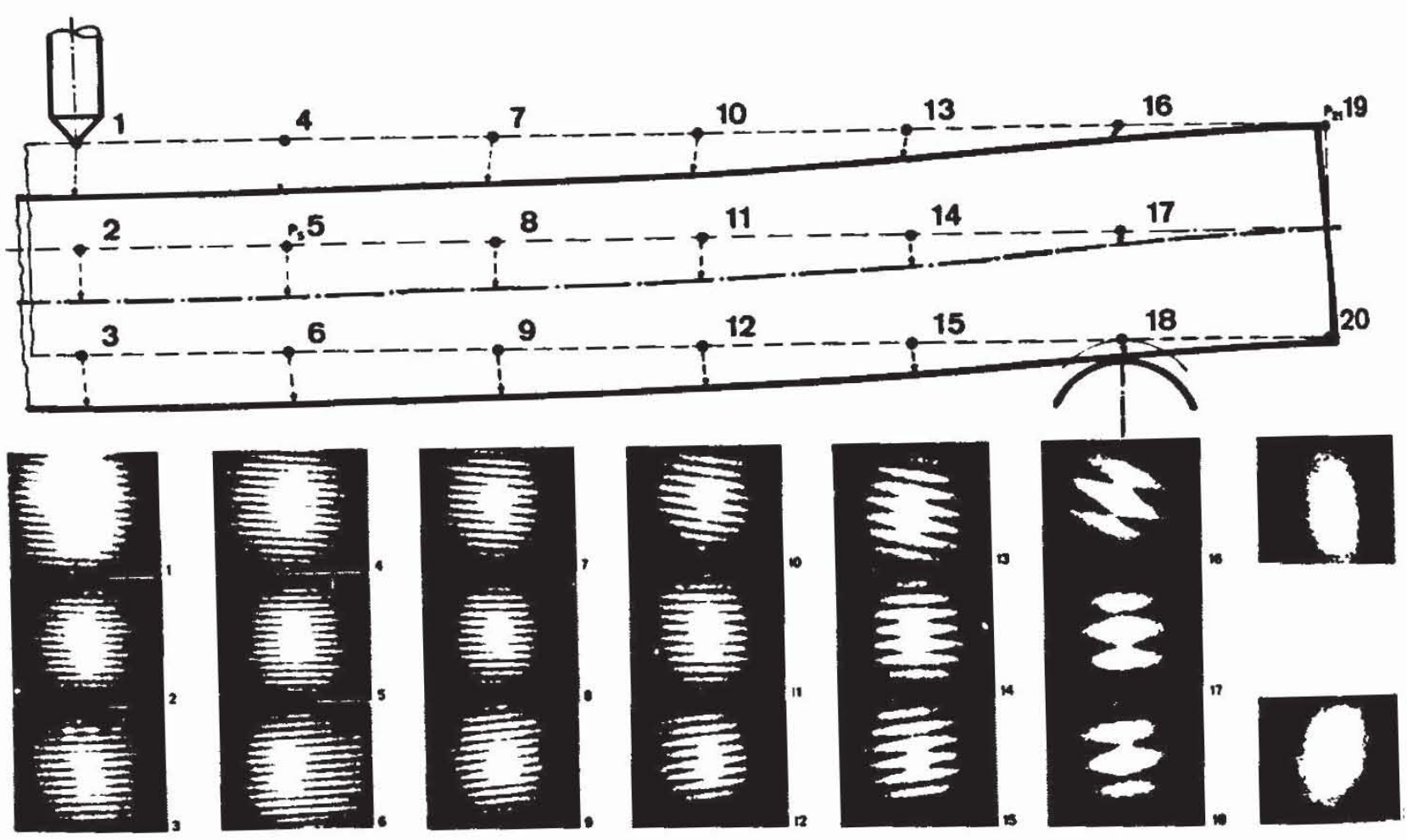

Fig. 5.7 Fringes obtained with a point-by-point analysis of a doubly exposed speckle pattern of a bending lever.

orientation, which are not stopped by the slit. Therefore the deformed regions will be visible irrespective of the nature of the deformations.

For a displacement much smaller than the speckle diameter, the method proposed by Leendertz $[5.8,5.10]$ may be appropriate. The positions of the speckles themselves remain basically unaltered-only their intensities change. Each time the optical path difference satisfies $2 \Delta \xi \sin \beta=n \lambda$ ( $n$ being an integer), the speckle becomes identical to the original.

\subsubsection{Time-Average Recordings}

For the study of harmonic oscillations, the speckle pattern is recorded by time-average exposure. Considering the oscillation of the object with $\rho \cos 2 \pi \nu t$, where $|\rho|$ is the amplitude of lateral oscillation in the object and $\nu$ is the frequency of oscillation, the complex amplitude in the Fraunhofer plane will be, according to Eq. (5-13),

$$
a_{1}\left(\mathbf{X}_{1}\right)=\iint\left\{T_{0}-b t_{0}\left\langle I\left(\mathbf{u}^{\prime}, \rho, t\right)\right\rangle\right\} \exp \left[-i 2 \pi \mathbf{u}^{\prime} \cdot \mathbf{X}_{1}\right] d \mathbf{u}^{\prime} .
$$

We again neglect the influence of an integration over a finite illuminated region of the recorded speckle pattern. It should, however, be noticed that for varying amplitude and direction of movement, regions of nearly constant movement need to be selected by an appropriately chosen dia- 
phragm. Substituting Eq. (5-11) into Eq. (5-16), again neglecting the undiffracted light and constants, we find

$$
\tilde{a}_{1}\left(\mathbf{X}_{1}\right)=\iint \psi^{\prime}\left(\mathbf{X}_{1}-\mathbf{X}_{\mathrm{q}}\right) \psi^{\prime *}\left(\mathbf{X}_{\mathrm{q}}\right) d \mathbf{X}_{\mathrm{q}} J_{0}\left[2 \pi \mathbf{X}_{1} \cdot \boldsymbol{\rho}^{\prime}\right]
$$

If the object roughness is of small scale, the scattered light will lead to a more uniform illumination of the pupil of the optical system and, therefore, the integration in the above expression can be extended over the entire exit pupil of the image-forming system. In the expression above, the autocorrelation of the exit pupil is modulated by a Bessel function of order zero and first kind. The intensity observed is

$$
\left|\tilde{a}_{1}\left(\mathbf{X}_{1}\right)\right|^{2}=\left|\iint \psi^{\prime}\left(\mathbf{X}_{1}-\mathbf{X}_{q}\right) \psi^{\prime *}\left(\mathbf{X}_{q}\right) d \mathbf{X}_{\mathrm{q}}\right|^{2} J_{0}^{2}\left(2 \pi \mathbf{X}_{1} \cdot \boldsymbol{\rho}^{\prime}\right) \text {. }
$$

The autocorrelation functions for an image-forming system working at numerical apertures $\sin \alpha^{\prime}=\mathbf{0 . 0 4 5}(\mathrm{f} / 2.8)$ and $0.011(\mathrm{f} / 11)$ are depicted in Fig. 5.8 for a stationary object, and in Fig. 5.9 for an oscillating object. The results were obtained by scanning the Fourier plane with a slit of width slightly larger than the speckle diameter determined by the diaphragm used to select the appropriate region of constant movement of the image. The slit was oriented parallel to the interference fringes and of width $0.1 \mathrm{~mm}$.

Ignoring the center due to the undiffracted light, gradual decrease of the autocorrelation function towards the edge of the exit pupil occurs for the stationary object (Fig. 5.8). The Bessel function $J_{0}\left(2 \pi \rho^{\prime} \cdot X_{1}\right)$ is multiplied by the autocorrelation function of the speckling in the pupil, which leads to the fringes in Fig. 5.9. The radius $r_{x_{1}}$, where the autocorrelation function of the pupil approaches zero, is given by $r_{x_{1}}=2 f_{1} \sin \alpha^{\prime}$, and consequently no fringes can be observed for $\left|x_{1}\right| \geq r_{x_{1}}$. Since $r_{x_{1}}$ is directly proportional to the numerical aperture $\left(\sin \alpha^{\prime}\right)$ of the image-forming optical system in the image space (Fig. 5.5), $\sin \alpha^{\prime}$ should be large (high F
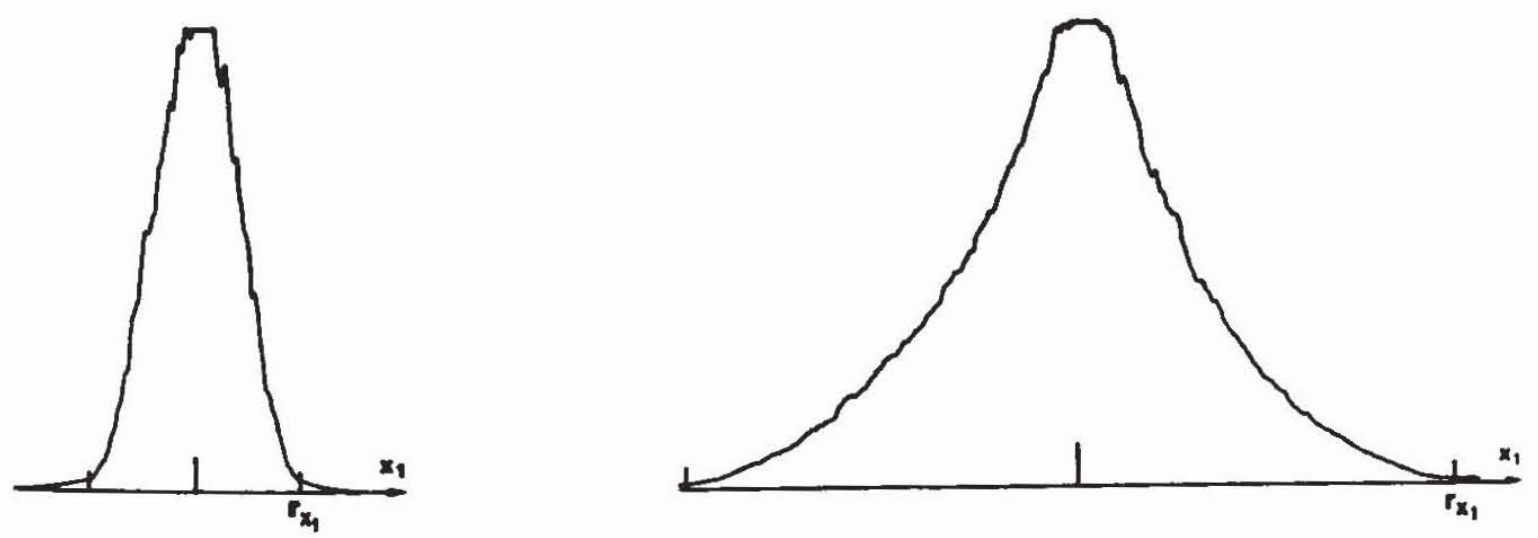

FIG. 5.8 Autocorrelation function for a stationary object recorded in the Fraunhofer plane with an image-forming lens of aperture $\mathrm{f} / 2.8$ (right) and $\mathrm{f} / 11$ (left). 

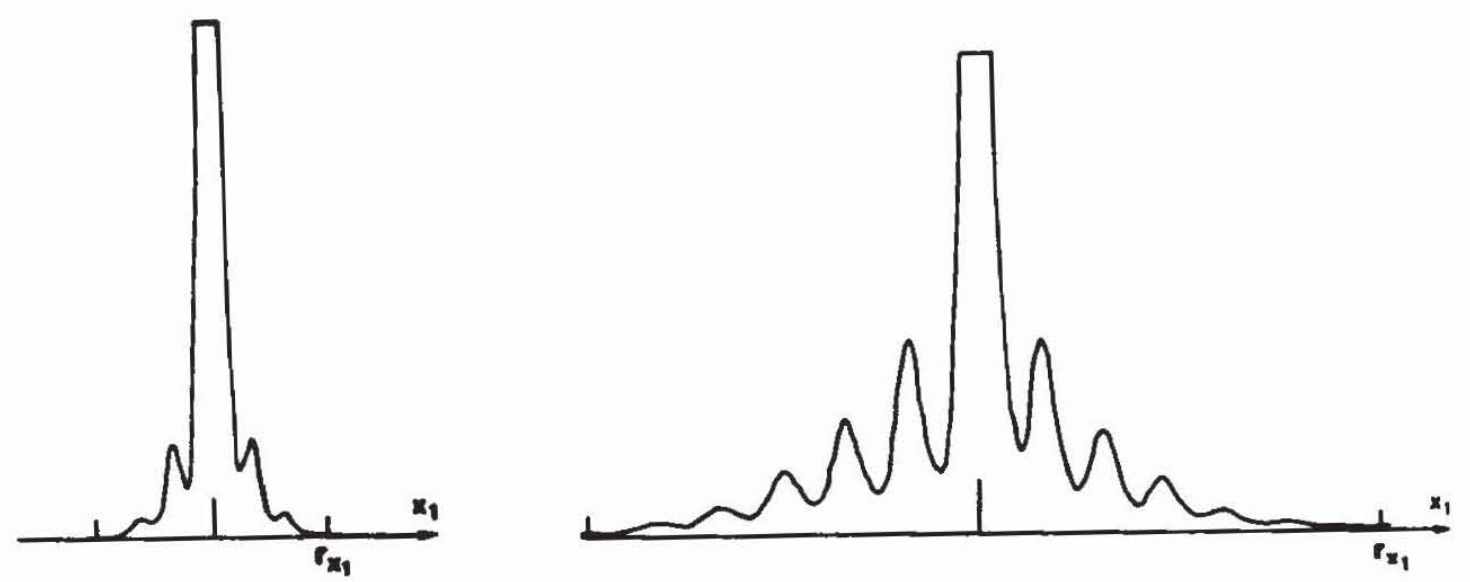

Fig. 5.9 Time-average intensity distribution for an oscillating object (amplitude $2 \rho_{0}=19$ $\mu \mathrm{m}$ ) recorded in the Fraunhofer plane with an image-forming lens of aperture $\mathbf{f} / \mathbf{2 . 8}$ (right) and $\mathrm{f} / 11$ (left).

ratio) to obtain a large number of fringes of good contrast in the Fraunhofer plane.

From Eq. (5-18) we obtain the minima of intensity for $2 \pi X \cdot \rho^{\prime}=2.40$, $5.52, \ldots$. Thus, denoting the separation of the first minima by $\left|x_{p_{1}}\right|$ and remembering the normalized coordinates defined in Eq. (5-5), we deduce that the amplitude of oscillation $\left|\rho_{0}^{\prime}\right|$ in the object is

$$
\left|\rho_{0}^{\prime}\right|=0.76\left(f_{1} \lambda\right) /\left(M\left|\mathbf{x}_{p_{1}}\right|\right),
$$

where $M$ is the lateral magnification of the image-forming optical system and $f_{1}$ is the focal length of the lens forming the Young's fringes. To improve the accuracy of the method, higher-order minima or maxima of $J_{0}$ can be evaluated. The orientation of the interference fringes is again perpendicular to the direction of the movement in the object.

To increase the sensitivity of the technique, the speckle pattern of the stationary object can be superposed on that of the oscillating object recorded in the time average. This leads to a slightly modified expression for the Young's fringes. The modified Eq. $(5-18)$ is

$$
\left|b\left(\mathbf{X}_{1}\right)\right|^{2}=\left|\iint \psi\left(\mathbf{X}_{1}+\mathbf{X}_{q}\right) \psi^{*}\left(\mathbf{X}_{\mathbf{q}}\right) d \mathbf{x}_{\mathbf{q}}\right|^{2}\left|K+J_{0}\left[2 \pi \rho^{\prime} \cdot \mathbf{X}_{1}\right]\right|^{2},
$$

where $K$ is a constant depending on the ratio of the exposure times of the stationary and oscillating speckle pattern. For $K \geq\left|K_{0}\right|$, when $\left|K_{0}\right|$ is the amplitude of the first minimum of $J_{0}$, the minima of $b\left(\mathbf{X}_{1}\right)^{2}$ occur for $J_{0}^{\prime}\left(2 \pi \rho^{\prime} \cdot X_{1}\right)=0$ and $J_{0}^{\prime \prime}>0$ and no longer for $J_{0}=0$, i.e., for $2 \pi \rho^{\prime} \cdot \mathbf{X}_{1}=$ $3.83,10.17, \ldots$. The spacing of the fringes can be increased in this way.

Different factors, such as nonlinearity of the photographic material and a limiting diaphragm for selecting the appropriate object region of almost 
constant movement, impair the fringe contrast. By an appropriate choice of $K$ it is possible to improve the contrast and sensitivity of the doubly exposed time-average recording of the speckle patterns slightly. The analysis can be extended to other types of movements, such as a continuous movement with constant velocity $v$. The square of the autocorrelation of the exit pupil will then be modulated by

$$
\left[\left(\sin \pi v t_{0}\right) /\left(\pi v t_{0}\right)\right]^{2},
$$

where $v$ is the reduced velocity and $t_{0}$ the exposure time.

\subsubsection{Comparison of Fringe Shapes for Different Motions}

Different types of fringes obtained from laser speckle photography will be summarized by using one-dimensional notation. In the previous analysis it was shown that a lateral in-plane shift leads to a displacement of the speckle pattern in the image plane by the same amount that the image of the object moves. By the double exposure technique the speckle pairs (original and shifted) lead to Young's cosine interference fringes in the Fraunhofer plane. The same fringe pattern is obtained by stroboscopic illumination of a harmonically oscillating object (see Fig. 5.10a). For harmonic oscillations, a probability density function can be considered, and for time-average exposures, this is of the form

$$
\operatorname{rect}(u / \rho) /\left[\pi\left(u^{2}-\rho^{2}\right)^{1 / 2}\right],
$$

as shown in Fig. 5.10b. The fringes obtained in the Fraunhofer diffraction pattern are proportional to the Fourier transform of the probability density function, leading to Bessel functions $J_{0}\left(2 \pi \rho X_{1}\right)$ of order zero and first kind. Superposition of the speckle pattern of a stationary object on that of the harmonically oscillating object recorded in the time average (see Fig. 5.10c) leads to

$$
\left[K+J_{0}\left(2 \pi \rho X_{1}\right)\right]
$$

For a continuous movement with velocity $v$, exposed in the time average, the probability density function is constant during the exposure time. The fringes obtained are of the form

$$
\left|\operatorname{sinc}\left(a X_{1}\right)\right|^{2}=\left|\left[\sin \left(\pi a X_{1}\right)\right] /\left(\pi a X_{1}\right)\right|^{2},
$$

where the total displacement $a=v t_{0}, t_{0}$ being the exposure time. The interference fringes are equivalent to the Fraunhofer diffraction pattern of a slit of width $a$ (Fig. 5.10d). For comparison, different Young's fringes obtained from speckle patterns of an oscillating object, recorded in the image plane, are depicted in Fig. 5.11. Figure 5.11A shows the fringes by 


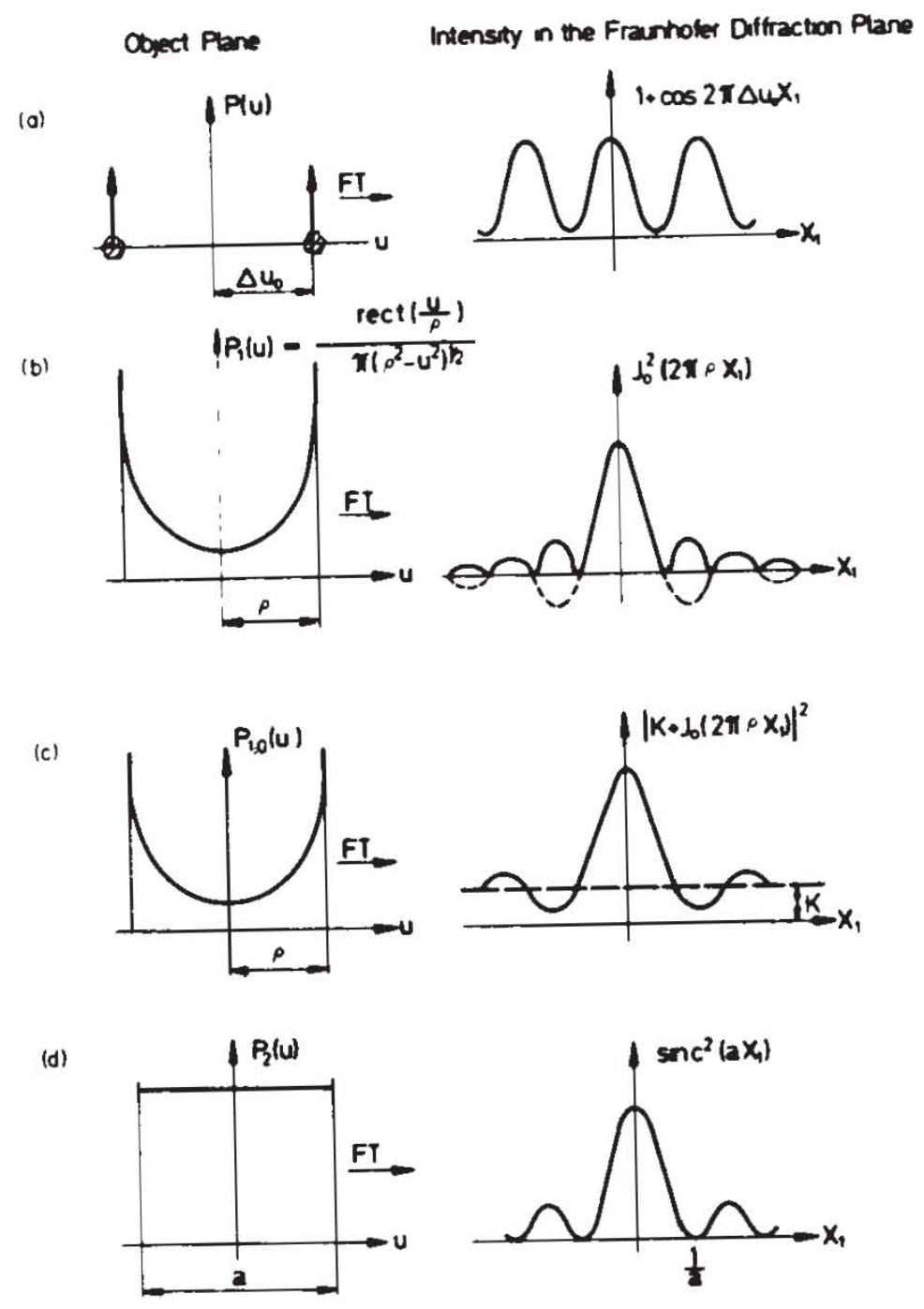

FIG. 5.10 Probability density functions of speckles and the expected Fraunhofer fringes for (a) displacement, (b) harmonic oscillation, (c) harmonic oscillation and superposition of a stationary object, and (d) a continuous movement.

stroboscopic illumination; the oscillating object was illuminated at the extreme positions only. By contrast, Fig. 5.11B shows the fringes of a time-average recording, and Fig. 5.11C those of the speckle pattern of the stationary object superposed on the time-average recording. Furthermore, an additional in-plane displacement of the oscillating object can be detected; Fig. 5.11D shows the Bessel function modulated by cosine fringes due to an additional displacement of $5 \mu \mathrm{m}$. Figure 5.12 illustrates Young's fringes for a continuous movement.

The analysis carried out so far applies to simple harmonic oscillation. The technique can be extended, however, to surfaces vibrating in two mutually perpendicular directions, and characteristic diffraction spectra can be obtained [5.16].

For a generalized motion of the object, the probability density function 

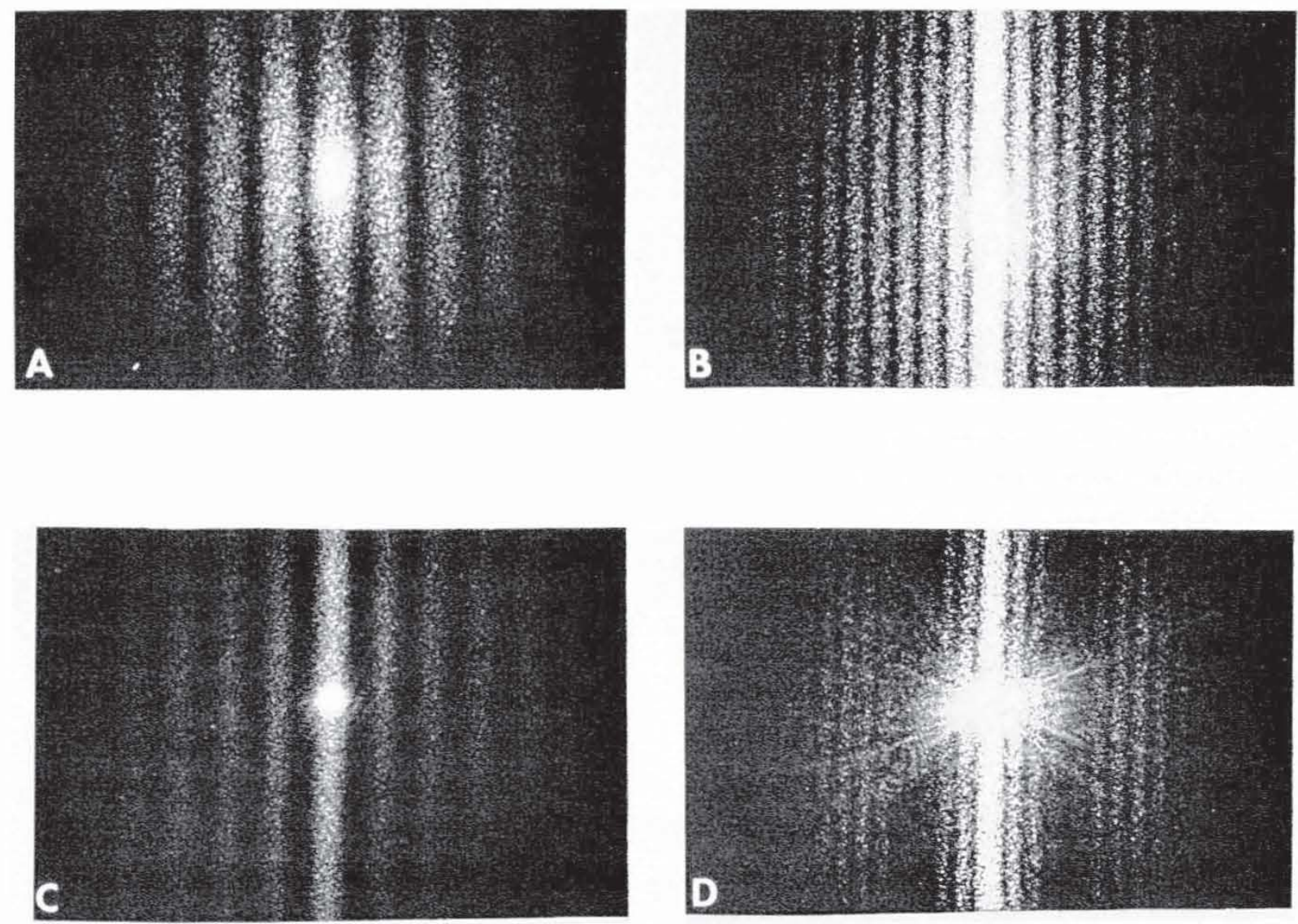

FIG. 5.11 Comparison of Young's fringes obtained from speckle patterns of a harmonically-oscillating object (amplitude of oscillation $2 \rho=19 \mu \mathrm{m}$ ) for (A) stroboscopic illumination, (B) a time-average recording, (C) a time-average recording with superposed speckle patterns of the stationary object, and (D) a time-average exposure of an oscillating object with an additional in-plane displacement of $5 \mu \mathrm{m}$.

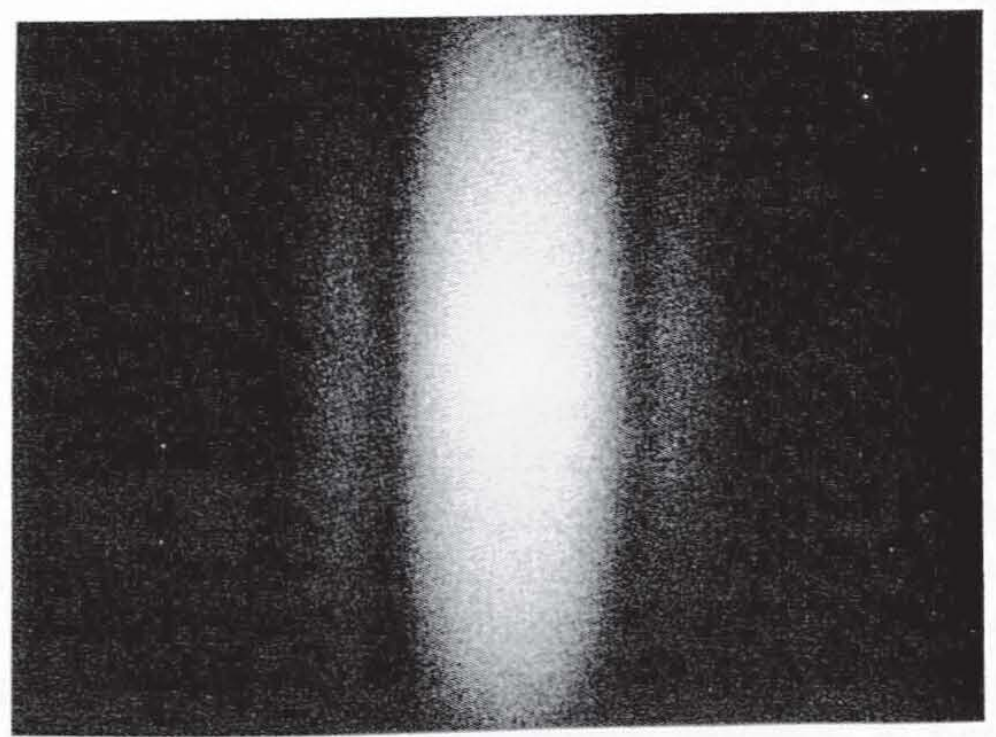

Fig. 5.12 Young's fringes from a speckle pattern of a time-average recording of a continuous movement with a velocity $v$. 
of the speckles may be complicated and its Fourier transform difficult to evaluate. An approximate solution can be found using the concept of stationary phase. It has been shown [5.16] that for some vibrations the main contribution to the diffraction spectrum comes from point sources located at the extreme positions of the movement. Orthogonal sinusoidal motion leads to the well-known Lissajous figures in the diffraction spectrum of the speckle patterns recorded in the time average.

In general, it is not possible to relate complicated diffraction patterns to the speckle trajectory that gives rise to it. The missing phase information required to reconstruct the motion can be supplied by recording an additional exposure of the stationary surface on the same frame, giving it a deliberate offset from the mean position of the time-averaged speckle pattern $[5.17,5.18]$. The diffraction spectrum from the resultant combination, when photographed, acts like a Fourier hologram from which two mirror images of the motion trajectory can be reconstructed, as discussed in detail in Chapter 7.

\subsection{Tilt Analysis by Fourier Transform Plane Recording}

As noted previously, the engineering measurement of tilt suggests that we record speckle patterns in the Fourier transform plane (Fraunhofer diffraction plane). Although there is no basic difference, we refer to the Fourier transform plane when the speckles of the object are recorded in the back focal plane of the lens, but to the Fraunhofer plane when the fringes are displayed. In this way superposed movements can be separated into out-of-plane tilts and in-plane movements (displacement and rotation). For intermediate recording planes the superposed movements cannot be easily separated. A tilt leads to a separation of identical speckle patterns in the Fourier transform plane of the object under test. An additional lateral shift leads to a phase shift in the Fourier transform, which has no consequences, at least for small in-plane movements, because only intensities are recorded.

Young's fringes for recorded speckle patterns of two superposed movements, one in-plane and the other a tilt perpendicular to it, are illustrated in Fig. 5.13. The speckle patterns were recorded in different planes, including the image and Fourier transform planes $(Z=0)$. The two movements can easily be separated by recording the speckle patterns for tilt in the Fourier transform plane and those for in-plane movements in the image plane. Combined movements observed in other recording planes are manifested in different spacings and orientations of the fringes obtained, for which the analysis of the separate movements becomes difficult. Double exposure techniques, or time-averaged recording for an 


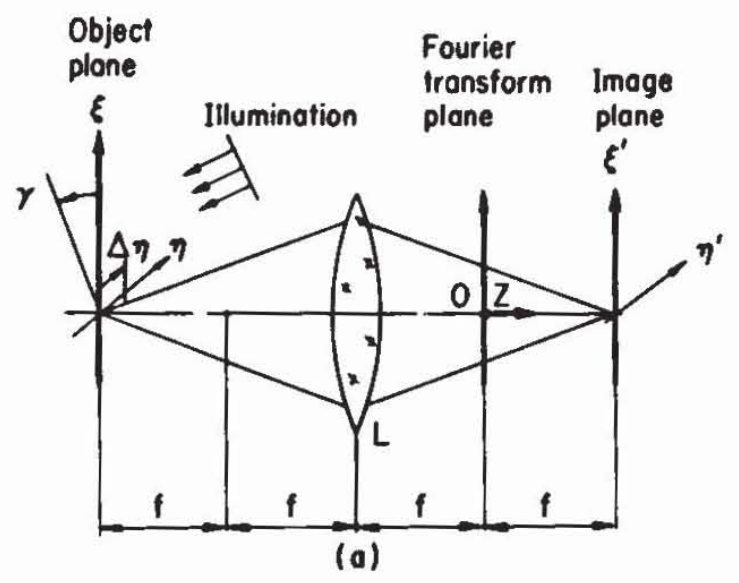

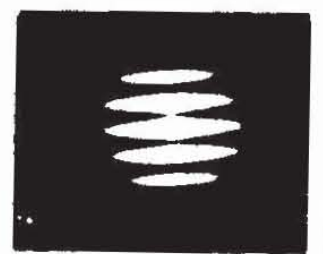

$Z=0$

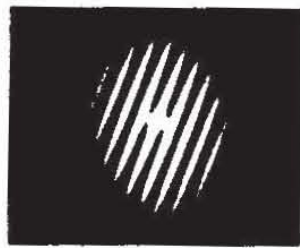

87

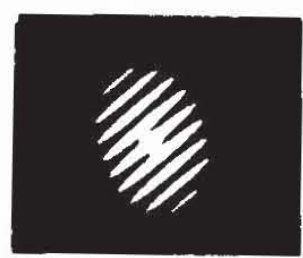

27

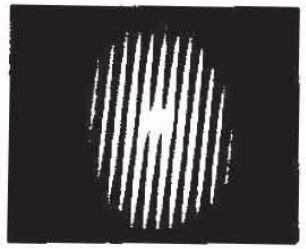

117

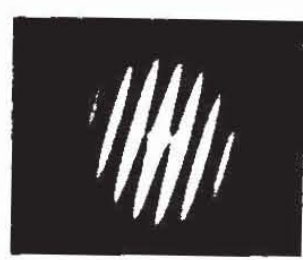

57

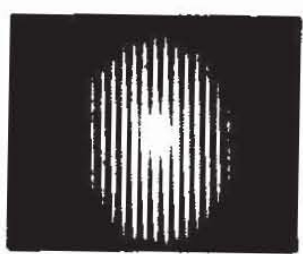

145.5

(b)

Fig. 5.13 (a) An experimental arrangement for obtaining the Young's fringes (b) from doubly exposed speckle patterns recorded with a tilt and an in-plane displacement (perpendicular to each other) as recorded in different planes from the Fourier transform plane $(Z=0)$ to the image plane $(Z=145.5)$.

oscillating object, can be applied in the Fourier transform plane in the same way as discussed previously for the image plane. In the present method, two or more practically identical speckle patterns of the optically rough object surface, before and after the tilts, are recorded in the Fourier transform plane of the object. Illuminating the developed photographic plate with a laser beam yields the Young's interference fringes with a spacing inversely proportional to the tilt angle. In the brief theory, presented below and supported by experimental data, we shall assume that the smallest tilt to be measured induces a shift of the speckles in the Fourier plane at least as large as the smallest speckle dimension, which is inversely proportional to the lateral size of the object field. In deriving the appropriate equations for measuring tilts, use has been made of the fact 
that the shift of the speckles in the Fourier transform recording plane is due to a tilt in the object plane [5.19].

\subsubsection{Two Successive Exposures}

For the analysis of tilts, a plane wave is preferred for illuminating the object. (With a spherical wave, as discussed in Chapter 8 and Ref. [5.20], the speckles should be recorded in the image of the light source.) According to Fig. 5.14, the Fourier transform of the complex amplitude of the object $A(\xi, \eta)$ is obtained in the back focal plane of the lens $L$. For convenience, the optically rough surface is placed in the front focal plane of the lens. Because only intensities are recorded, the position of the object is not critical for the experiments.

Illuminating the optically rough surface with a plane wave having an angle of incidence $\beta$ (Fig. 5.14), the Fourier transform is

$$
a(\mathbf{x})=\iint A(\xi) \exp [(i 2 \pi \xi \cdot \mathbf{x}) /(\lambda f)] d \xi,
$$

where: $\boldsymbol{\xi}, \mathbf{x}$ are vectors representing the coordinates $(\xi, \eta)$ in the object plane and $(x, y)$ in the Fourier transform plane; $\lambda$ is the wavelength; and $f$ is the focal length of lens $L . A(\xi)$ is the complex amplitude immediately after the surface, namely,

$$
A(\xi)=\Phi(\xi) \exp [i 2 \pi \xi \cdot \beta / \lambda] .
$$

The complex amplitude of the optically rough surface at a point $P(\xi)$ may be written, in accordance with Section 5.3.1, as the superposition of a set of waves:

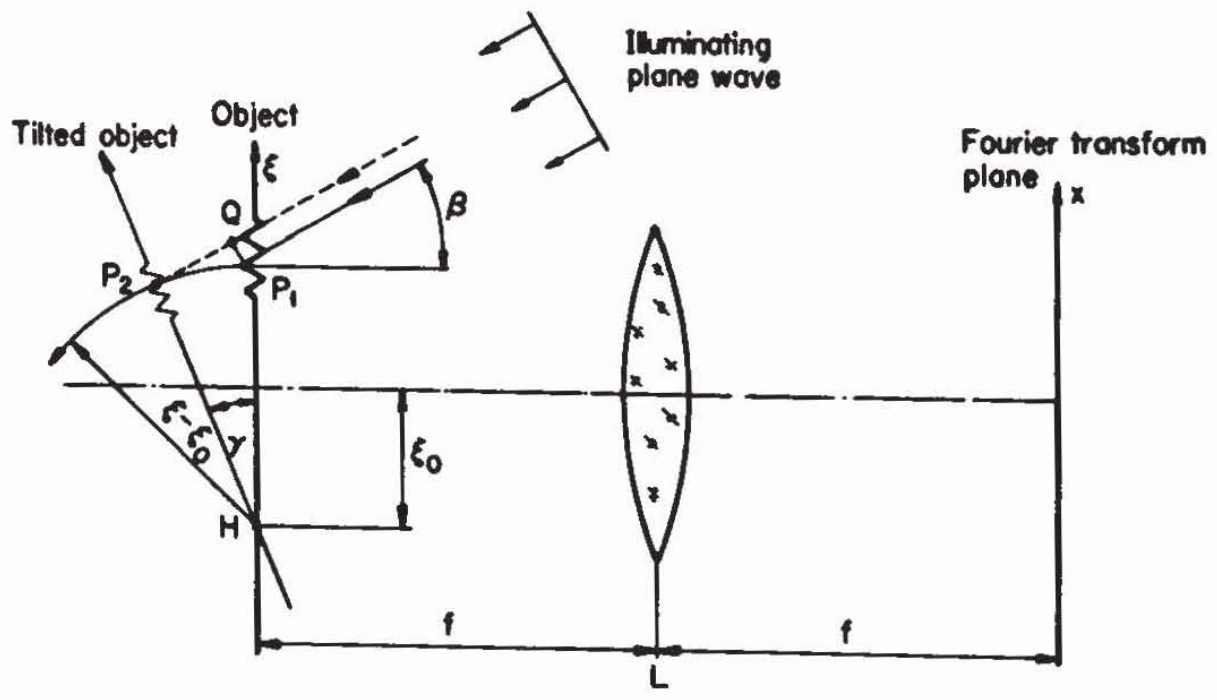

FIG. 5.14 Experimental setup for measuring tilts by recording speckle patterns in the Fourier transform plane. 


$$
\Phi(\xi)=\sum_{\mathbf{p}} \psi\left(\mathbf{x}_{p}\right) \exp \left[i 2 \pi \mathbf{x}_{p} \cdot \xi / \lambda f\right] .
$$

This may be written as an integral in the limit to represent the distribution of complex amplitudes as a function of $\boldsymbol{\xi}$ leading to a Fourier integral. $\boldsymbol{\xi}, \mathbf{x}_{p}$ are vectors representing the two-dimensional coordinates $(\xi, \eta)$ in the object plane and $\left(x_{p}, y_{q}\right)$ in the Fourier transform plane.

To simplify the writing, one-dimensional notation is used for the following development. The theory can, however, easily be extended to two dimensions. Furthermore, reduced coordinates are introduced, namely $X_{0}=-x /(\lambda f)$. Substituting Eq. (5-22) into Eq. (5-21) gives

$$
a\left(X_{0}\right)=\int_{-\infty}^{\infty} \Phi(\xi) \exp [(i 2 \pi / \lambda) \xi \sin \beta] \exp \left[-i 2 \pi \xi X_{0}\right] d \xi .
$$

If the object is tilted by an angle $\gamma$, as shown in Fig. 5.14, the wavefront immediately after the surface will be modified. For this purpose the optical path difference of an identical point on the optically rough surface, before and after the tilt, needs to be found. A plane wave is very convenient for tilt analysis because the optical path difference is a function of $\beta$ and the tilt $\gamma$ only. In Fig. 5.14, the center of rotation is assumed to be at $H$. The identical point $P_{1}$ on the rough surface moves to $P_{2}$, introducing an optical path change into the incident oblique wave:

$$
\left[Q P_{2}\right]=2\left(\xi-\xi_{0}\right) \sin (\gamma / 2) \cos [\beta-(\gamma / 2)] \text {. }
$$

The refractive index is considered to be $n=1$. For small angular tilts $\gamma$, $\left[Q P_{2}\right] \approx\left(\xi-\xi_{0}\right) \gamma \cos \beta$. As a result, the original identical wavefront $A(\xi)$ will be modified to become

$$
\begin{aligned}
A_{1}(\xi)= & \Phi(\xi) \exp [(i 2 \pi / \lambda) \xi \sin \beta] \exp [(i 2 \pi / \lambda) \xi \gamma] \\
& \times \exp \left[(i 2 \pi / \lambda)\left(\xi-\xi_{0}\right) \gamma \cos \beta\right] .
\end{aligned}
$$

The spectrum of the original identical speckle pattern $\varphi\left[X_{0}-(\sin \beta / \lambda)\right]$ in the Fourier transform plane is therefore shifted and can be written as

$$
a\left(X_{0}, \gamma\right)=\varphi\left[X_{0}-\frac{\sin \beta}{\lambda}-\frac{\gamma}{\lambda}(1+\cos \beta)\right] \exp \left[\frac{i 2 \pi}{\lambda} \xi_{0} \gamma \cos \beta\right] .
$$

An additional in-plane lateral shift leads to a phase term in the Fourier transform plane. Since we record intensities, a small lateral shift will have no consequences on the speckles recorded but may reduce the correlation of the speckles or the contrast of the fringes obtained. The sum of the resulting intensities of the speckle patterns, recorded before and after a tilt $\gamma$, will be, by neglecting constants, $i\left(X_{0}, \gamma\right)=\left|a\left(X_{0}\right)\right|^{2}+$ $\left|a\left(X_{0}, \gamma\right)\right|^{2}$, leading to 


$$
i\left(X_{0}, \gamma\right)=\left|\varphi\left[X_{0}-\frac{\sin \beta}{\lambda}\right]\right|^{2}+\left|\varphi\left[X_{0}-\frac{\sin \beta}{\lambda}-\frac{\gamma}{\lambda}(1+\cos \beta)\right]\right|^{2},
$$

which can be written as

$$
\begin{aligned}
i\left(X_{0}, \gamma\right)= & \left|\varphi\left(X_{0}\right)\right|^{2} *\left\{\delta\left(X_{0}-\frac{\sin \beta}{\lambda}\right)\right. \\
& \left.+\delta\left[X_{0}-\frac{\sin \beta}{\lambda}-\frac{\gamma}{\lambda}(1+\cos \beta)\right]\right\},
\end{aligned}
$$

where $*$ is, as customary, convolution, and $\delta$ is the delta function.

The linear region of the amplitude transmission curve of the photographic material is selected, and the undiffracted light, as well as constants, are neglected. The amplitude in the Fraunhofer plane is the Fourier transform of the intensity of the speckle patterns recorded by double exposure, namely,

$$
a_{1}\left(x_{1}, \gamma\right)=\int_{\text {aperture }} i\left(X_{0}, \gamma\right) \exp \left[i 2 \pi f x_{1} X_{0} / f_{1}\right] d X_{0},
$$

where $x_{1}=$ rectangular coordinate in the Fraunhofer plane, and $f, f_{1}$ are (respectively) the focal lengths of the lens system used to perform the Fourier transform of the object and to display the fringes in the Fraunhofer plane. By substituting Eq. (5-28) into Eq. (5-29), the intensity in the Fraunhofer plane is

$\left|a_{1}\left(x_{1}, \gamma\right)\right|^{2}=2\left|\operatorname{FT}\left[\left|\varphi\left(X_{0}\right)\right|^{2}\right]\right|^{2}\left\{1+\cos \left[(2 \pi f) /\left(\lambda f_{1}\right) x_{1} \gamma(1+\cos \beta)\right]\right\}$.

As the result of a tilt, the original speckles are shifted in the Fourier transform plane. These pairs of speckles lead to Young's fringes in the Fraunhofer plane by illuminating the developed plate. The spacing of the fringes is inversely proportional to the tilt angle in the object. From the width of the cosine fringes $x_{c}$ obtained in the Fraunhofer plane of the coherently illuminated developed photographic plate, the tilt in the object is found to be

$$
\gamma=\left(f_{1} / f\right)\left\{\lambda /\left[(1+\cos \beta) x_{\mathrm{c}}\right]\right\} .
$$

Only the intensity of the fringes in the Fraunhofer plane is observed. Therefore, different experimental arrangements can be considered for displaying the Young's fringes. In a configuration such as in Fig. 5.6b, $f_{1}$ in Eq. (5-31) needs to be replaced by the distance between the plate and focal plane. In Eq. (5-29) the integration is supposed to be extended from $-x$ to $+x$. In practice, however, the integration will only be extended over the illuminated part of the recorded speckle pattern. Because the 
region of almost equal tilt needs to be selected on the object, the field aperture for displaying the Fraunhofer fringes can be increased, which reduces the speckle size in the fringe plane. The speckle size in the Fraunhofer plane should not exceed a third of the fringe spacing for the observation of reasonable fringe contrast.

So far, the cosine fringes of Eq. (5-30) have been discussed. The first term, describing the optically-rough surface, which was mathematically expressed in Eq. (5-23) by the superposition of spherical waves, will now be considered. Equation (5-23) leads to an intensity in the spectrum of

$$
\left|\varphi\left(X_{0}\right)\right|^{2}=\sum_{p} \sum_{q} \psi\left(X_{p}\right) \psi^{*}\left(X_{q}\right) \delta\left(X_{0}-X_{p}\right) \delta\left(X_{0}-X_{q}\right),
$$

resulting in a set of speckle patterns for which the size of the speckles is determined by the interference of the waves coming from the edges of the limiting object or of the illuminated field, whichever is smaller. The Fourier transform of $\left|\varphi\left(X_{0}\right)\right|^{2}$ leads to the autocorrelation of the optically rough surface $\int \Phi\left(\mathrm{x}_{1}+\xi\right) \Phi^{*}(\xi) \delta \xi$. The Young's fringes are therefore multiplied by the square of the autocorrelation of the object field or the illuminated area on the optically rough surface as the case may be. A typical set of Young's fringes resulting from doubly exposed speckle patterns with a tilt of $\gamma_{\xi}=60$ seconds introduced between two exposures is shown in Fig. 5.15A. Introducing two tilts $\left(\gamma_{\eta}=60\right.$ seconds and $\gamma_{\eta}=60$ seconds) perpendicular to each other in succession with the appropriate exposures before and after the tilts, creates the fringes of Fig. 5.15B. Since tilts were introduced in two directions orthogonal to each other, two sets of fringes are displayed in the Fraunhofer diffraction pattern. For the experiments the spectra of the object, an aluminum plate illuminated with an 18-mW He-Ne laser at $\beta=45$ degrees, were recorded on Agfa Scientia 8E75 plates. For displaying the fringes in the Fraunhofer plane a $\mathrm{He}-\mathrm{Ne}$ laser was used, but fringes can also be seen in white light. Measurements related to superposed movements indicate that an additional in-plane movement does not impair the results for tilt measurements.

\subsubsection{Multiple Successive Exposures}

Burch and Tokarski [5.21] have studied successive exposures by recording multiple exposures of the speckles in the image of a ground glass, displacing the photographic plate between exposures. Similar results can be arrived at when the photographic plate is exposed $N+1$ times with $N$ tilts of $\gamma$ between.

The recorded intensity in the Fourier transform plane is, according to Eq. (5-28), 

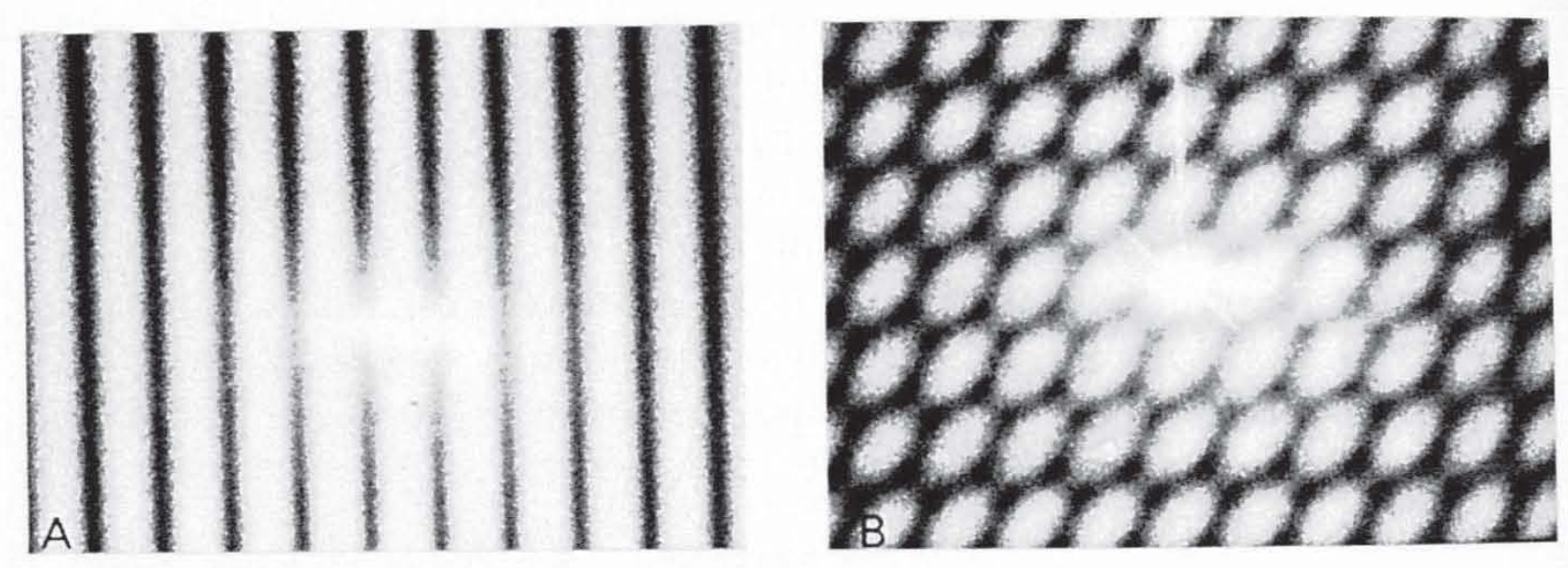

FIG. 5.15 The Young's fringes obtained from doubly exposed speckle patterns $\left(\beta=45^{\circ}\right)$, with (A) a tilt of $60^{\prime \prime}$ introduced between the two exposures, and (B) two tilts, both of $60^{\prime \prime}$ and perpendicular to each other, introduced between the three exposures.

$$
i_{2}\left(X_{0}, \gamma\right)=\left|\varphi\left(X_{0}\right)\right|^{2} * \sum_{n=0}^{1} \delta\left\{X_{0}-\frac{\sin \beta}{\lambda}-\frac{n \gamma}{\lambda}(1+\cos \beta)\right\},
$$

where $n=0,1,2, \ldots, N$. The intensity of the coherently illuminated developed plate in the Fraunhofer plane can be written, neglecting constants, according to Eq. (5-30) as

$$
\begin{aligned}
\left|a_{2}\left(x_{1}, \gamma\right)\right|^{2}= & \left|\int \Phi\left(x_{1}+\xi\right) \Phi^{*}(\xi) \delta \xi\right|^{2} \\
& \times\left|\sum_{n=0}^{N} t_{n} \exp \left[\left(i 2 \pi n \gamma x_{1} / \lambda\right)\left(f / f_{1}\right)(1+\cos \beta)\right]\right|^{2}
\end{aligned}
$$

Assuming all the exposures are equal $\left(t_{n}=t\right)$ and the object is given equal tilts $\gamma$ between exposures, we obtain the following result by summing the geometrical series in Eq. (5-34):

$$
\begin{aligned}
\left|a_{2}\left(x_{1}, \gamma\right)\right|^{2}= & \left|\int \Phi\left(x_{1}+\xi\right) \Phi^{*}(\xi) \delta \xi\right|^{2} \\
& \times\left\{\frac{\sin \left[\left(\frac{N+1}{2}\right) \frac{2 \pi}{\lambda} x_{1} \frac{f}{f_{1}} \gamma(1+\cos \beta)\right]}{\sin \left[\frac{\pi}{\lambda} x_{1} \frac{f}{f_{1}} \gamma(1+\cos \beta)\right]}\right\}^{2} .
\end{aligned}
$$

This is the familiar expression for the interference pattern from a set of $N+1$ identical equidistant pinholes of equal transmission. In between the main maxima there are $N-1$ equally spaced secondary maxima considerably weaker than the main ones. The secondary maxima can be suppressed by taking the exposure times in the ratio of binomial coefficients.

In Fig. 5.16 fringes of six equal tilts of $\gamma_{\xi}=30$ seconds between the seven exposures are reproduced. The secondary maxima between the main ones are typical of multiple beam fringes. 

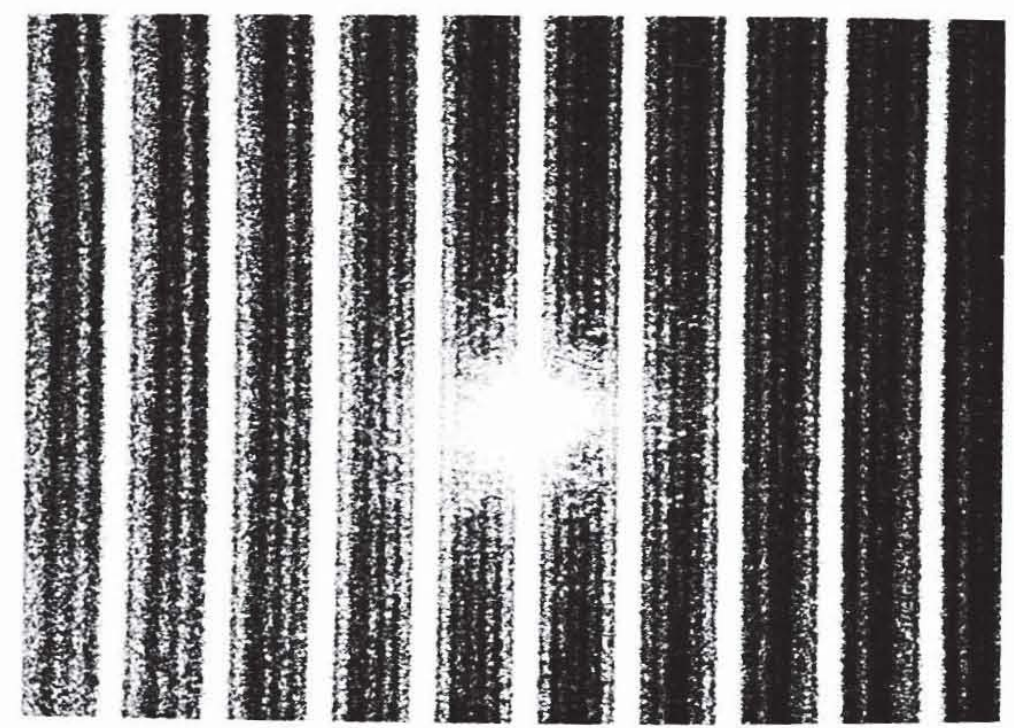

FIG. 5.16 Fringe pattern obtained from a multiply exposed speckle pattern as recorded in the Fourier transform plane for six equal tilts of $30^{\prime \prime}$ between seven exposures $\left(\beta=45^{\circ}\right)$.

\subsubsection{Two Successive Exposures with Two Beam ILLUMINATION}

In the previous discussion the object was illuminated by a single plane wave. Using two beam illumination, with the waves incident at angles $\beta_{1}$ and $\beta_{2}$ to the perpendicular of the object [5.19], similar results are obtained. The Young's fringes will, however, be modulated. The intensity in the Fraunhofer plane can be found approximately, by neglecting constants, to be

$$
\begin{aligned}
\left|a_{1}\left(x_{1}\right)\right|^{2} \approx & 4\left|\int \Phi\left(x_{1}+\xi\right) \Phi *(\xi) \delta \xi\right|^{2} \\
& \times\left\{1+\cos \left[\left(\frac{2 \pi f}{\lambda f_{1}} x_{1} \gamma\right)\left(1+\frac{\cos \beta_{1}+\cos \beta_{2}}{2}\right)\right]\right. \\
& \left.\times \cos \left[\left(\frac{2 \pi f}{\lambda f_{1}} x_{1} \gamma\right)\left(\frac{\cos \beta_{1}-\cos \beta_{2}}{2}\right)\right]\right\},
\end{aligned}
$$

where $\int \Phi\left(x_{1}+\xi\right) \Phi^{*}(\xi) \delta \xi$ indicates the autocorrelation of the object field studied. The cosine fringes, similar to those of Eq. (5-30), are therefore modulated by

$$
\cos \left(\frac{2 \pi f}{\lambda f_{1}} \gamma x_{1}\right)\left(\frac{\cos \beta_{1}-\cos \beta_{2}}{2}\right) .
$$

For $\beta_{1}= \pm \beta_{2}$ the same results as for one beam illumination are obtained. Typical results, with incidence angles $\beta_{1} \neq \beta_{2}$, are shown in Fig. 5.17, where the additional modulation of the Young's interference fringes can 

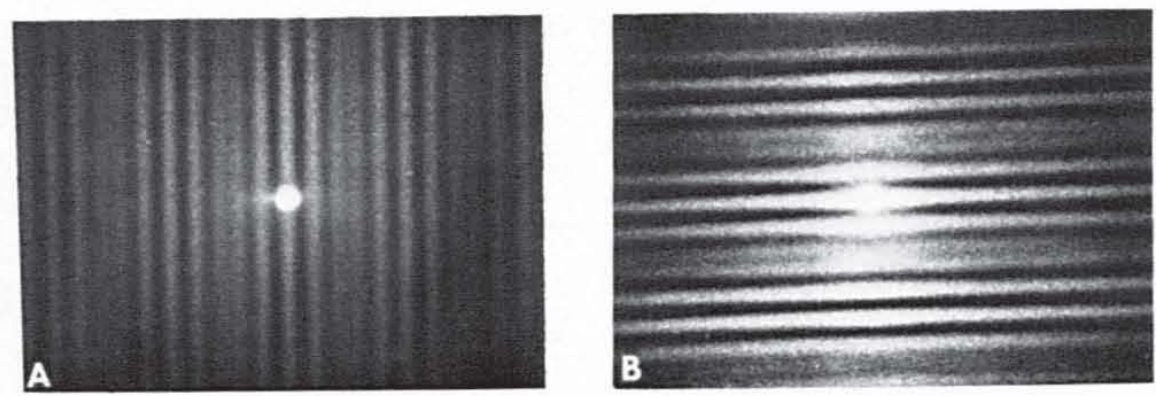

FIG. 5.17 Photographs of the fringes obtained using a ground glass as the object and two illuminating beams with unequal angles of incidence $\left(\beta_{1}=30^{\circ}, \beta_{2}=55^{\circ}\right)$ for a doubly exposed speckle pattern with a tilt (A) of $\gamma_{\xi}=60^{\prime \prime}\left(\gamma_{\xi}=\gamma\right.$ in Fig. 5.14), and (B) of $\gamma_{\eta}=60^{\prime \prime}$ perpendicular to $\gamma_{\xi}$.

be seen. In Fig. 5.18 the inverse of the fringe spacing is plotted against accurately measured tilt angles introduced for one and two beam illumination. To illustrate that tilt measurements can be obtained very accurately, even in the presence of in-plane lateral displacements, an additional lateral in-plane movement of $40 \mu \mathrm{m}$ was introduced, leading to a linear phase in the Fourier transform plane. It has no consequences when intensities are recorded, and the corresponding experimental results are marked by $O$ in Fig. 5.18. To obtain fringes of good contrast, the surface grain of the object should be fine enough to spread the light over an adequate area in the Fourier transform plane. A decrease in contrast is obtained for a

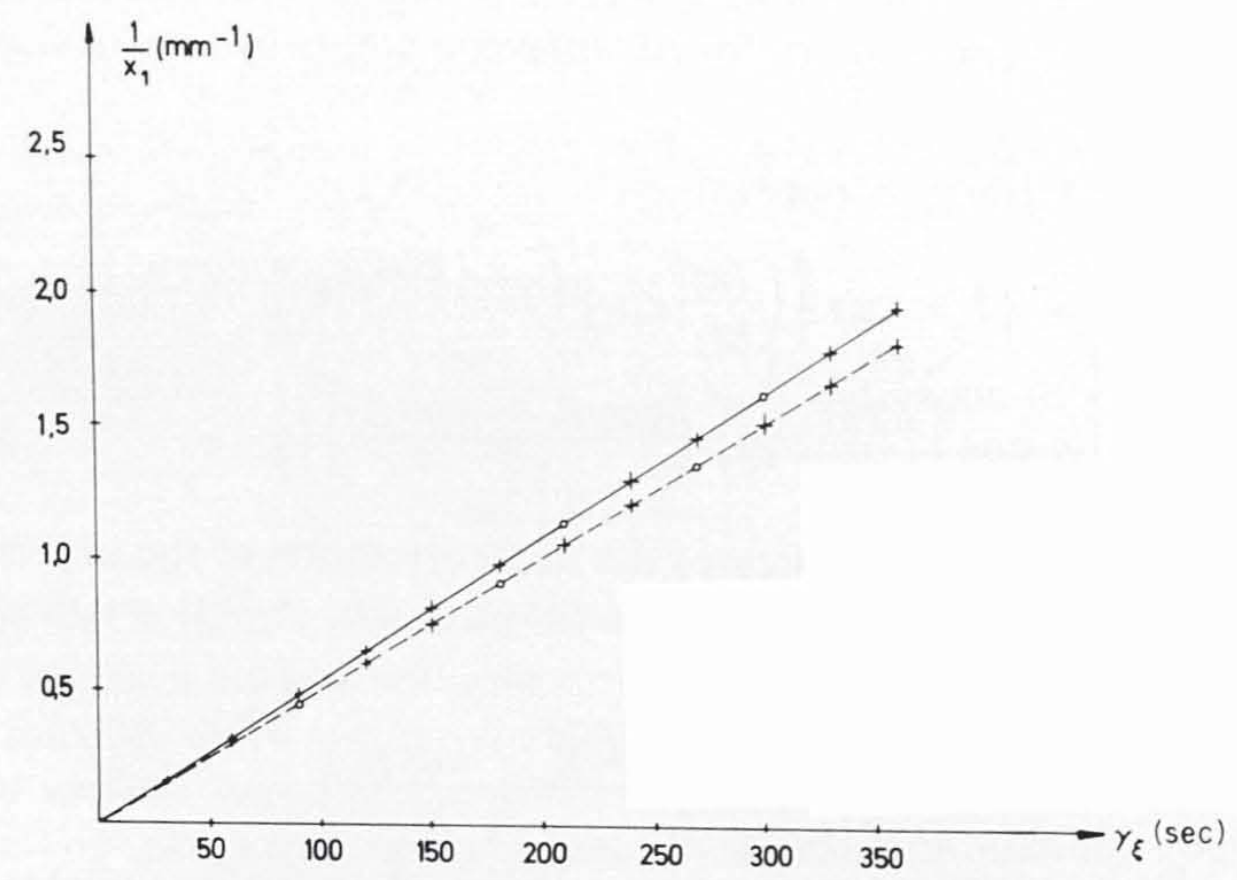

FIG. 5.18 Experimental results (+) showing the inverse fringe spacing as plotted against the measured tilts, for comparison with the theoretical results for $(\cdots) \beta=50^{\circ}$ and $(-\cdots)$ $\beta_{1}=30^{\circ}, \beta_{2}=55^{\circ}$. (The experimental results marked by $\bigcirc$ indicate the measurements taken with an additional lateral shift of $40 \mu \mathrm{m}$ ). 
coarse surface (very coarse ground glass). If the light is multiple scattered, for example by translucent surfaces, the speckle pattern becomes noncorrelated and no fringes are visible. Stetson [5.22] has pointed out that the recording of speckle grains in tandem, at different focal planes in the image space, provides a method for extracting strains, shears, and rotation in addition to displacements.

\subsection{Limitations of Speckle Photography for Engineering Measurements}

In the previous sections it was shown that the speckle patterns are recorded in the Fourier transform plane (or the image of the point source according to Gregory [5.20]) for tilt, and in the image plane for in-plane displacement and deformation measurements. Tilts introduced into the object between two exposures lead to a shift of the identical speckle patterns in the entrance pupil of the image-forming optical system. Inplane displacements give a phase shift in the entrance pupil and a lateral shift of identical speckles in the image. Since only intensities are recorded, tilts do not directly impair the accuracy of in-plane measurements. In-plane displacement does not reduce the accuracy of tilt measurements for small movements, but reduces the contrast of the fringes due to some decorrelations of the speckle pattern.

In the analysis of superposed movements by speckle photography, tilts are a severe restriction, especially when a small aperture is needed for in-plane measurements in the presence of in-line-of-sight movements (parallel to the optical axis). Movements parallel to the line of sight also reduce the correlation of the speckle patterns. In addition, scattering from the photographic grains will cause a reduction in fringe contrast, particularly towards the edge of the diffraction halo.

The lower limit for in-plane and tilt analysis is given by the width of individual speckles, i.e., the limiting aperture. The movement needs to be larger than the speckle size in the appropriate direction. For high speckle correlation, i.e., good fringe contrast, an accuracy of $\sigma / 50$ can be obtained, where $\sigma$, the speckle size in the appropriate plane, can be as small as $1 \mu \mathrm{m}$. The maximum displacement for tolerable fringe definition for an optical system working at $\mathrm{f} / 4$ is about $0.3 \mathrm{~mm}$ with unit magnification. Larger displacements can be measured with an appropriate demagnification.

\subsubsection{Movements Parallel to The Line-of-Sight}

Small displacements parallel to the line-of-sight (parallel to the optical axis) do not appreciably influence the speckle patterns. To ensure that the 
speckles remain practically unaltered, the change of focus between two exposures should not exceed the Rayleigh limit, i.e., the path difference at the edge of the pupil should not exceed $\lambda / 4$. Hence (Fig. 5.5)

$$
\Delta \zeta^{\prime} \leq \lambda / 2 \sin ^{2} \alpha^{\prime} \quad \text { and } \quad \Delta \zeta \leq \lambda / 2 \sin ^{2} \alpha .
$$

Low-contrast fringes can still be observed by exceeding the range of depth by a factor of $2 . \Delta \zeta$ is the defocusing in the object plane and $\Delta \zeta^{\prime}$ in the image plane. $\alpha, \alpha^{\prime}$ are the aperture angles in the object and image space, respectively. This implies that for measurements where a large defocusing may occur in the presence of other movements, the numerical aperture of the image-forming optical system should be small. In speckle photography, however, high-aperture lenses are very often necessary for the detection of superposed movements with tilts or when small in-plane movements need to be studied. Larger motions (in the line-of-sight) can, however, be tolerated for a demagnified image of the object.

\subsubsection{Limits on Tilts for in-Plane Measurements}

A tilt in the object leads to a lateral shift of an identical speckle in the entrance pupil. The shifted speckle should, however, remain inside the entrance pupil. Otherwise the corresponding speckle pairs are no longer correlated (Fig. 5.5). In other words, the optical path difference imparted to a particular speckle by an additional tilt $\gamma$ should be smaller than $\lambda$, from which it follows that

$$
\Delta \gamma_{0}<\lambda /[(1+\cos \beta) \sigma] \text { or } \quad \Delta \gamma_{0}<\theta_{0} /(1+\cos \beta),
$$

where $\sigma$ is the speckle size and $\theta_{0}$ is the angle of the illuminated pupil seen from the object. Furthermore, a tilt $\gamma$ imparts an additional phase shift to an individual speckle already laterally shifted by $\Delta \xi$. To obtain good correlation of the speckles recorded in the image plane, leading to high fringe contrast, the total optical path difference should not much exceed $\lambda / 4$. For an optical path difference of $\lambda$, the fringe correlation will be nearly zero, and no fringes appear. For good fringe contrast

$$
\Delta y \leq \lambda /[4(\sigma+\Delta \xi)(1+\cos \beta)]
$$

where $\beta$ is the angle of incidence of the light. The tolerance on tilt angle is thus inversely proportional to $\Delta \xi$ and proportional to the numerical aperture of the image-forming system. The tolerance on tilt angle seems to be the most severe limitation to speckle applications by photographic recording. It becomes especially acute when low-aperture imaging is attempted at large demagnification in the presence of movements parallel to the optical axis. To verify the model used for obtaining limits on tilts when in- 
plane movements are measured, experiments were carried out for superposed movements with deliberately changed parameters. The interference patterns displayed in the Fraunhofer plane of the speckle patterns were directly scanned by a slit in front of a photomultiplier. The fringe contrast, defined as

$$
c=\left(I_{\max }-I_{\min }\right) /\left(I_{\max }+I_{\min }\right),
$$

is plotted in Fig. 5.19 as a function of the introduced tilt $\gamma$. The experimental data were: $\lambda=632.8 \mathrm{~nm} ; \Delta \xi=0.025 \mathrm{~mm} ; f=156 \mathrm{~mm} ; \beta=30$ degrees; $M=1: 1$; and pupil diameter-45 mm. It was found that Eq. (5-34) agrees well with the experiments. For an optical path difference $\leq \lambda / 4$ excellent fringe contrast results, but for a path difference $\geq \lambda$ the contrast vanishes. On this basis, tilt limits for in-plane displacement can be found.

A further limitation of speckle applications is multiple scattering of the light. On a similar model we can find the decorrelation of the speckles for large in-plane movements on tilt measurements. For most practical applications they do not seriously impair the fringe contrast.

\subsubsection{Coherence of the Laser Source}

The brightness of an individual speckle is dependent only on the relative phase of light vectors scattered from individual points on the surface giving rise to the speckle. It is, of course, independent of the absolute phase of the light reaching the surface. Therefore, a constant phase change of the incident illuminating wave introduced between exposures in
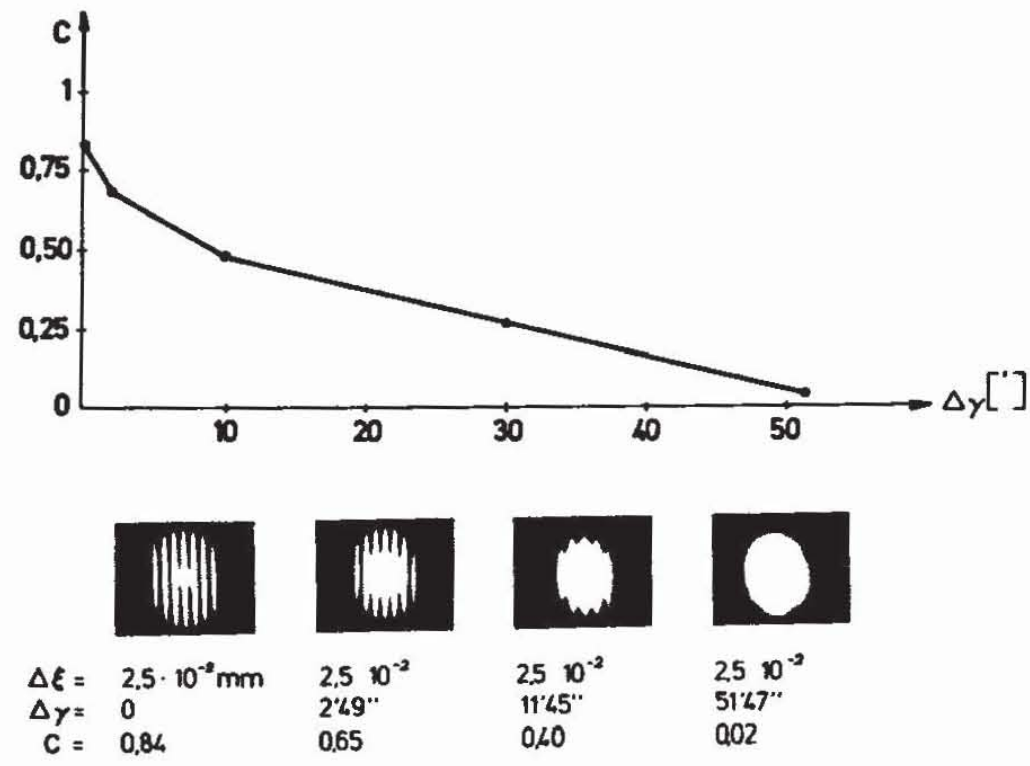

Fig. 5.19 Contrast reduction in the fringes as a result of object tilt, as obtained from measurement of the in-plane movements. 
double-exposure recording has no consequences, provided there are no local variations. The laser radiation must, however, be such that both temporal and spatial coherence are maintained over at least the dimensions of an individual speckle. This is not difficult to achieve, even when using a crude pulsed laser source [5.23].

\subsection{Discussion}

To apply holography for studying three-dimensional movements, with tilts in the presence of lateral motion for instance, requires an elaborate analysis and determination of the appropriate plane of localization of the interference fringes [5.24]. These problems do not occur in the application of speckling to studying three-dimensional movements. In addition, no reference wave is needed. Furthermore, stability does not appear to be a problem. The method will therefore be a very useful engineering tool. Speckle photography for studying movements parallel to the optical axis can also be applied, but its practical use for engineering applications is limited.

\subsubsection{Tilt and Deformation Measurement}

The experimental arrangement for recording data is very simple. The object is illuminated with coherent light and, in a typical double exposure sequence, it is in different states for each of the two exposures. By illuminating the developed plate in reflection or transmission with a point source, such as a penlight, several orders of Young's fringes, with spacing and direction perpendicular to the displacement of the selected region of the specimen, will be seen. Emulsions used should have a high modulation transfer function (MTF). For good sensitivity the Agfa Scientia 10E75 or 10 E56 plates (and occasionally films) are often used, depending on the wavelength. Plate and film processing follow standard practice, and bleaching of the plates may be useful in improving the diffraction efficiency, especially when low-power lasers are used for data processing. Best exposure of the plates or films seems to be that giving an overall intensity transmittance of approximately $50 \%$ unbleached. In the doubleexposure technique, each exposure is theoretically one-half of the total required; in practice it is slightly more.

In order to extract the displacement data from the doubly exposed plate, a simple optical processing system is used (Fig. 5.6). The sensitivity of the method can be altered primarily by changing the focal length of the lens displaying the fringes in the Fraunhofer plane or the distance between photographic plate and Fraunhofer plane (depending on the arrangement 
for displaying the fringes). It also depends on the magnification of the image-forming lens or its focal length when speckle patterns are recorded in the Fourier transform plane. Furthermore, a data processor [5.25] that selects given fringes by a blocking spatial filter can be used. A general conclusion is that the balance between sensitivity and range must be reached for any given measurement problem. These two parameters can be established to some extent after some preliminary data recording. For in-plane deformations with displacements varying across the surface of the object, a point-by-point illumination of the speckle pattern can be applied. By noting the fringe spacing and orientation, both the magnitude and orientation of the object movement can be recorded. For the analysis of three-dimensional motions of the object, a second photograph of the same object, or part of it, taken in the Fourier transform plane will be required.

Archbold and Ennos have applied laser photography to measuring the deformation of weld cracks under load [5.26]. The technique proved to be simple, and could be carried out on the machine without the need for dismantling. The sensitivity was found to be around ten times higher than for a conventional moiré technique. In addition, speckle photography can be applied successfully under difficult environmental conditions. For example, it has been employed to monitor the distortion occurring around a simulated crack in a large steel pressure vessel, as described by Gregory in Chapter 8. Luxmoore, in Chapter 9, describes the measurement of crystal length changes during annealing. Furthermore, plastic materials having relatively low moduli of elasticity seem to be very appropriate for laser speckle applications, and complete strain fields can be very accurately plotted over the surface of elastic materials.

Speckle pattern photography can also be applied to the study of phase objects. For this purpose, speckle patterns are photographed through a phase object. The individual speckles are displaced over varying distances depending upon phase irregularities in the object. A double exposure is made with the object only being present during one of the two exposures [5.27, 5.28].

\subsubsection{Vibration MEASUREMENT}

As already indicated, speckling has proved to be a very powerful tool for vibration analysis, and for some applications is even more appropriate than holographic techniques. More often, however, it supplements holography. The experimental arrangement does not need to be as stable as for holography, and the system is applicable to a larger range of vibration amplitudes. 
The real time analysis of vibrations is fast and practical for engineering applications. Time-average holography and electronic speckle pattern interferometry provide fringe patterns that represent vibration amplitudes distributed across the object, and the phase deformation may be revealed if either the object illumination or the reference wave is phase modulated [5.29]. The resulting fringe pattern is then determined by the relative motion between the object and the phase-modulating device. In this way, contours of constant phase can be quickly and easily obtained by means of a time-averaged electronic speckle pattern interferometer, as discussed in Chapter 6. A similar result can be reached by stroboscopic illumination: however, the experimental setup is more complicated.

Speckle photography for analyzing mechanical vibrations in the presence of superposed movements becomes simple as long as the tilts are small. Speckle patterns are recorded by the time-average methods in either the image or Fourier transform plane. In one application, tuning forks and quartz crystals used as frequency normals in electronic watches were successfully analyzed and adjusted [5.30]. As an example, consider the tuning fork shown in Fig. 5.20. It is a symmetric configuration, driven by electromagnetic heads $\left(P_{1}\right.$ and $\left.P_{2}\right)$. The tuning fork was constructed so as to be insensitive to shocks, and independent of gravity. Its frequency of

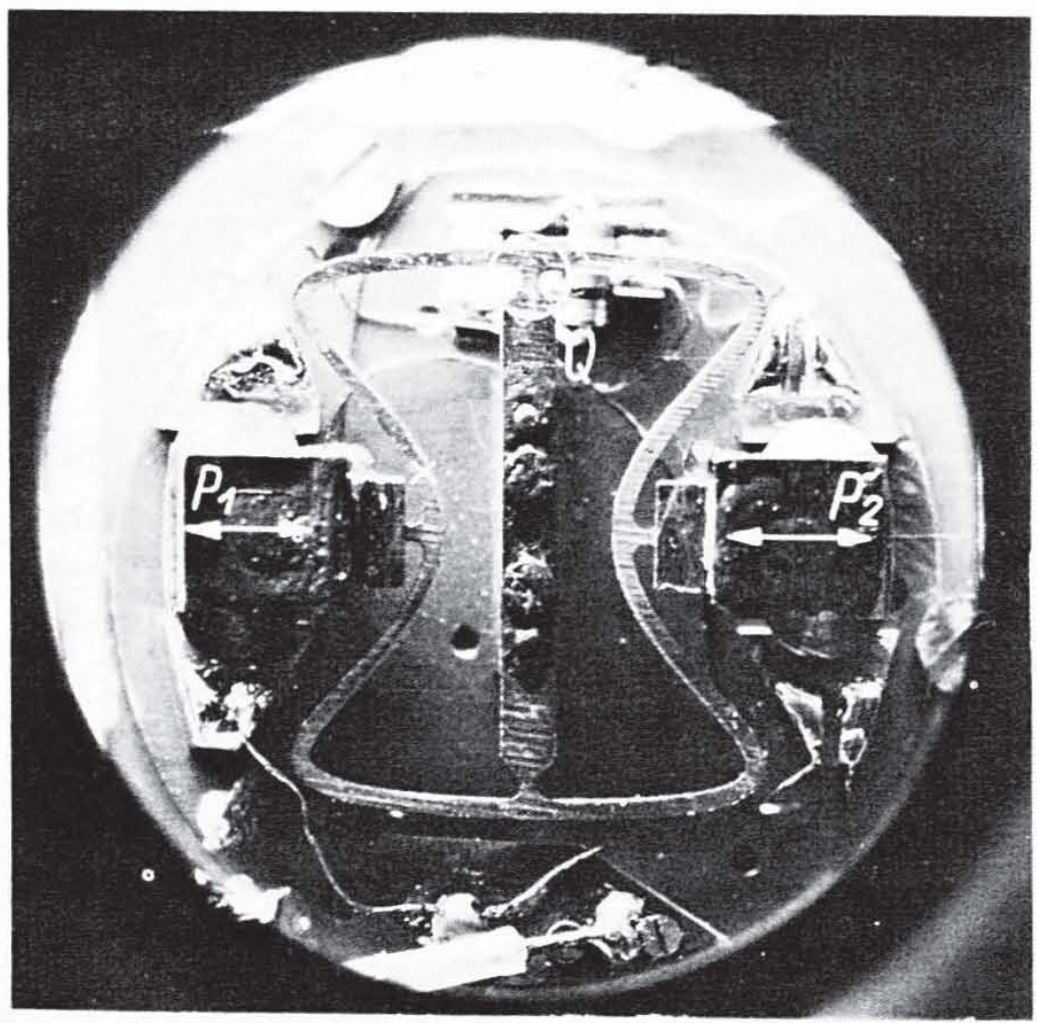

FiG. 5.20 Photograph of the tuning fork. with an oscillation frequency of $1015 \mathrm{~Hz}$. from an electronic watch. 
oscillation is $1015 \mathrm{~Hz}$. When an electric field is applied, the fork oscillates and $\mathrm{Q}$ remains stationary. The tuning fork is $20 \mathrm{~mm}$ long, $0.8 \mathrm{~mm}$ wide, and is driven with $10 \mu \mathrm{W}$. An interference pattern obtained by a holographic technique, using stroboscopic illumination, is shown in Fig. 5.21. Because of superposed movements, the fringes obtained were not localized in one plane, and the interpretation became rather difficult for an engineering application.

Speckling proved very useful for this vibration analysis application. A time-average speckle pattern of the oscillating tuning fork recorded in the image plane was illuminated by a converging wave. The autocorrelation of the pupil is modulated by a Bessel function accounting for in-plane oscillations (Fig. 5.22A). For measuring the out-of-plane movements (tilts), speckle patterns were recorded in the Fourier transform plane. A typical photograph of fringes obtained in this manner is presented in Fig. 5.22B.

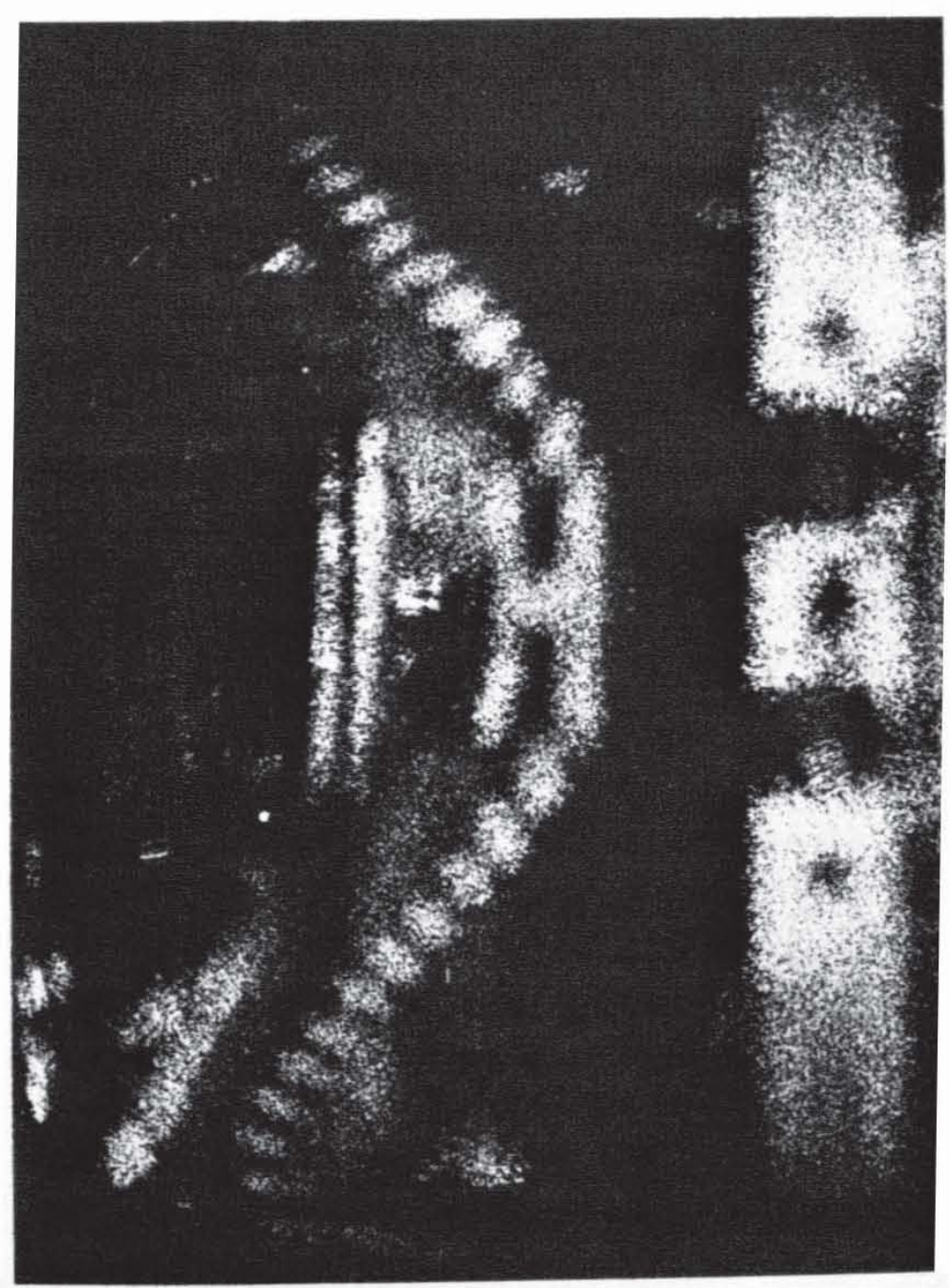

FIG. 5.21 Interference fringes of the oscillating tuning fork as obtained holographically with stroboscopic illumination. 

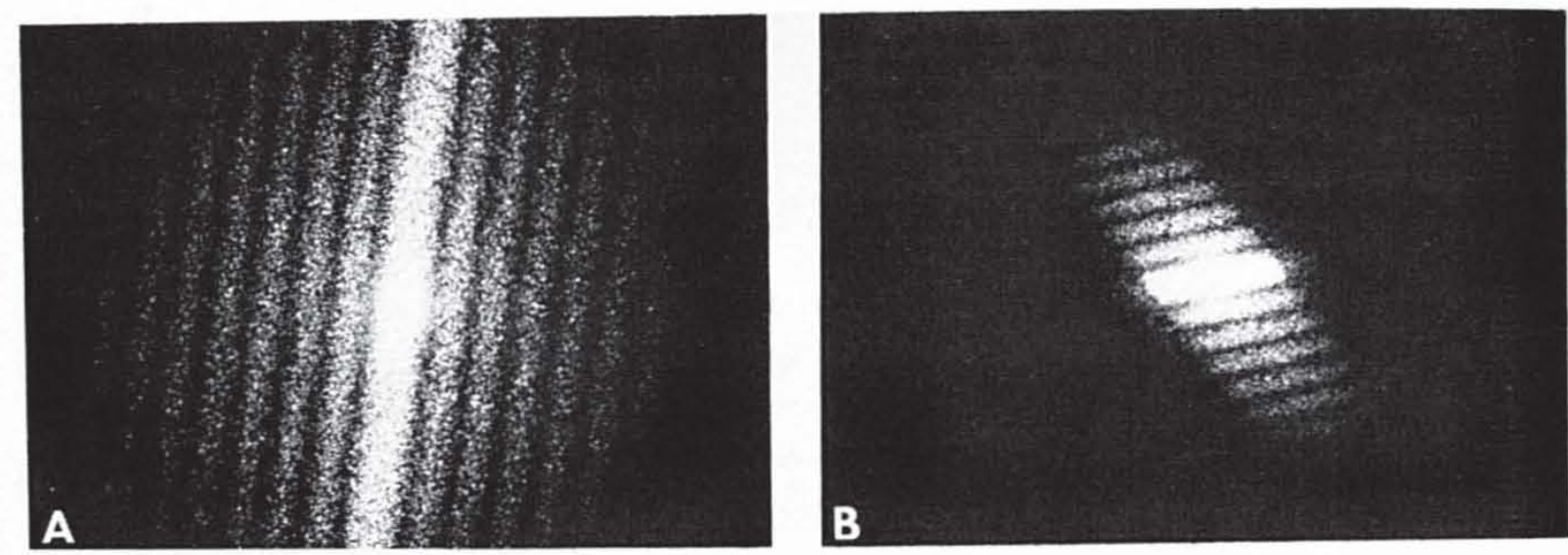

FIG. 5.22 Fringes recorded in the Fraunhofer plane from time-average speckle patterns of the oscillating tuning fork recorded in (A) the image plane, and (B) the Fourier transform plane. (The region of interest is identified in Fig. 5.20 by an X).

In-plane displacements and tilt components of motion can easily be obtained for different regions of the tuning fork. Speckle photography, therefore, provided a means for monitoring the design and adjustment for minimizing tilts, for instance. In Fig. 5.23 the amplitudes of oscillation of a tuning fork are plotted against the voltages applied for both (a) in-plane movements and (b) tilts. Joyeux and Lowenthal [5.31] have developed a method for measuring amplitudes of in-plane vibrations, ranging from angstroms to microns, by a generalized moiré method in real time.

\subsubsection{Deformation and White Light Speckle Photography}

Laser speckle photography is not always applicable to very large objects, and fails where the surface is subject to excessive tilts. For some

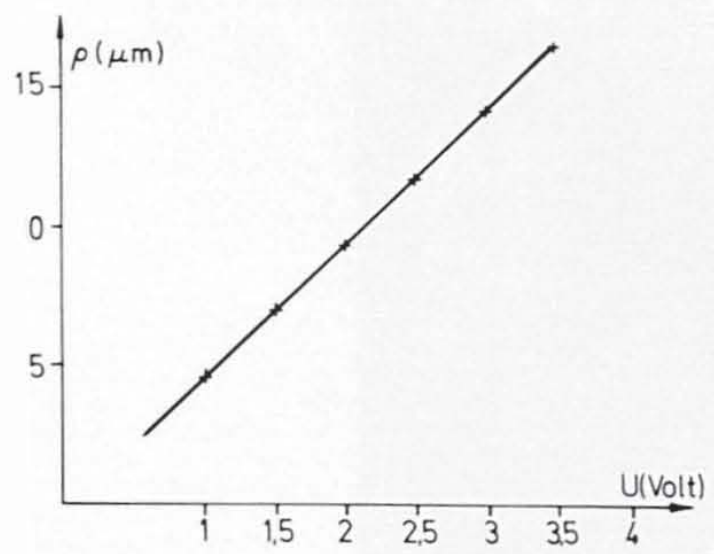

(a)

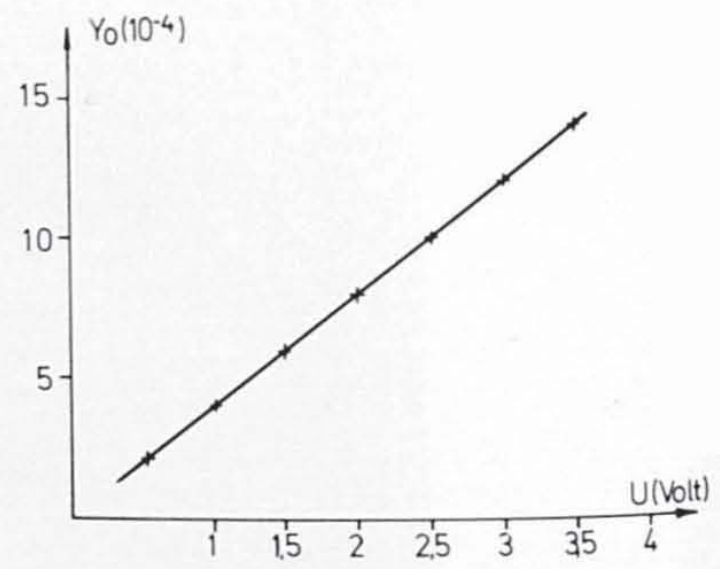

(b)

FIG. 5.23 The amplitude of oscillation of the tuning fork as determined by speckle photography, and plotted as a function of the voltage applied, for (a) in-plane oscillation, and (b) tilt. 
applications the white light speckle technique, discussed in Chapter 9 can be applied. In addition, Burch and Forno [5.32] have proposed a highsensitivity moiré. grid technique for studying deformations on large objects illuminated with white light. The basic experimental setup is similar to that used in laser speckle photography. The technique applies particularly to very large objects, relating the results directly to deformation. Therefore, for many applications of speckle photography a laser is not necessary either for recording the speckles or for displaying Young's fringes.

In conclusion, it appears that speckle techniques for deformation measurement and vibration analysis will be developed further. The results obtained are generally easy to interpret, compared with those from holographic interferometry, and the mathematics involved is not difficult. Finally, the equipment necessary for laser speckle photography is not unduly complicated.

\section{References}

5.1. E. Archbold, A. E. Ennos, and P. A. Taylor, A laser speckle interferometer for the detection of surface movements and vibration, Proc. ICO, Reading, 1969, Vol. 8 (J. Home Dickson, ed.). Oriel Press, Newcastle-upon-Tyne, 1970.

5.2. L. Ek and N. E. Molin, Detection of the nodal lines and the amplitude of vibration by speckle interferometry, Opt. Commun. 2, 419 (1971).

5.3. B. Eliasson and F. M. Mottier, Determination of the granular radiance distribution of a diffuser and its use for vibration analysis, J. Opt. Soc. Amer. 61,559 (1971).

5.4. K. A. Stetson, New design for laser image-speckle interferometer, Opt. Laser Technol. 2, 179 (1970).

5.5. J. M. Burch, Interferometry with scattered light, "Optical Instruments and Techniques" (J. Home Dickson, ed.), p. 213. Oriel Press, Newcastle-upon-Tyne, 1970.

5.6. K. A. Stetson, Holographic vibration analysis, in "Holographic Nondestructive Testing" (R. K. Erf, ed.). Academic Press, New York, 1974.

5.7. B. Eliasson and R. Dändliker, Contrast of photographically recorded speckle patterns, Opt. Acta 23, 813 (1976).

5.8. J. A. Leendertz, Measurement of surface displacement by interference of specklepatterns, Proc. ICO, Reading 1969, Vol. 8 (J. Home Dickson, ed.). Oriel Press, Newcastle-upon-Tyne, 1970.

5.9. G. Groh, Engineering uses of laser produced speckle patterns, Proc. Symp. Engineering Uses Holograph. Cambridge Univ. Press, London, 1970.

5.10. J. A. Leendertz, Interferometric displacement measurement on scattering surfaces utilizing speckle effect, J. Phys. E: Sci. Instrum. 3, 214 (1970).

5.11. H. J. Tiziani, Application of speckling for in-plane vibration analysis, Opt. Acta 18, 891 (1971).

5.12. H. H. Hopkins, Canonical pupil coordinates in geometrical and diffraction image theory, Proc. Conf. Photogr. Spectros. Opt., Tokyo, 1964. Japanese Journal of Applied Physics 4, Supplement I, 1965.

5.13. H. H. Hopkins and H. J. Tiziani, Speckling in diffraction patterns and optical images formed with the laser, Proc. Int. Symp. Hologr., Besançon, 1970. 
5.14. U. Köpf, A coherent optimal method for contactless measurements of local displacements and vibrations, Optik 35, 144 (1972).

5.15. G. N. Watson, "A Treatise on the Theory of Bessel Functions"' (2nd ed.). Cambridge Univ. Press, London and New York, 1966.

5.16. E. Archbold and A. E. Ennos, Two-dimensional vibrations analyzed by speckle photography, Opt. Laser Technol. 7, 17 (1975).

5.17. A. W. Lohmann and G. P. Weigelt, The measurement of depth motion by speckle photography, Opt. Commun. 17, 47 (1976).

5.18. A. W. Lohmann and G. P. Weigelt, Speckle methods for the display of motion paths, J. Opt. Soc. Amer. 66, 1271 (1976).

5.19. H. J. Tiziani, A study of the use of laser speckle to measure small tilts of optically rough surfaces accurately, Opt. Commun. 5, 271 (1972).

5.20. D. A. Gregory, Basic physical principles of defocused speckle photography: a tilt topology inspection technique, Opt. Laser Technol. 8, 201 (1976).

5.21. J. M. Burch and J. M. J. Tokarski, Production of multiple beam fringes from photographic scatterers, Opt. Acta 15, 101 (1968).

5.22. K. A. Stetson, Problem of defocusing in speckle photography, its connection to hologram interferometry, and its solutions, J. Opt. Soc. Amer. 66, 1267 (1976).

5.23. A. E. Ennos, Speckle interferometry, in "Laser Speckle and Related Phenomena" (J. C. Dainty, ed.). Springer-Verlag, Berlin and New York, 1975.

5.24. K. A. Stetson, Fringe interpretation for hologram interferometry of rigid-body motions and homogeneous deformations, J. Opt. Soc. Amer. 64, 1 (1974).

5.25. E. Archbold and A. E. Ennos, Displacement measurement from double-exposure laser photographs, Opt. Acta 19, 253 (1972).

5.26. E. Archbold and A. E. Ennos, Laser photography to measure the deformation of weld cracks under load, Nondestruct. Test. 8, 181 (1975).

5.27. S. Mallick and M. L. Roblin, Speckle pattern interferometry applied to the study of phase objects, Opt. Commun. 6, 45 (1972).

5.28. U. Köpf, Application of speckling for measuring the deflection of laser light by phase objects, Opt. Commun. 9, 374 (1973).

5.29. O. J. Lokberg and K. Høgmoen, Vibration phase mapping using electronic speckle pattern interferometry, Appl. Opt. 15, 2301 (1976).

5.30. H. J. Tiziani, Optische Methoden zur Schwingungsanalyse der Stimmgabel einer elektronischen Uhr, Optik 34, 442 (1972).

5.31. D. Joyeux and S. Lowenthal, Real time measurement of Angström order transverse displacement or vibrations by use of laser speckle, Opt. Commun. 4, 108 (1971).

5.32. J. M. Burch and C. Forno, A high sensitivity moiré grid technique for studying deformation in large objects, Opt. Eng. 14, 178 (1975). 